\title{
Fractional Sobolev's Spaces on Time Scales via Conformable Fractional Calculus and Their Application to a Fractional Differential Equation on Time Scales
}

\author{
Yanning Wang, Jianwen Zhou, and Yongkun Li \\ Department of Mathematics, Yunnan University, Kunming, Yunnan 650091, China \\ Correspondence should be addressed to Yongkun Li; yklie@ynu.edu.cn
}

Received 23 June 2016; Revised 26 August 2016; Accepted 8 September 2016

Academic Editor: Pietro d'Avenia

Copyright (C) 2016 Yanning Wang et al. This is an open access article distributed under the Creative Commons Attribution License, which permits unrestricted use, distribution, and reproduction in any medium, provided the original work is properly cited.

Using conformable fractional calculus on time scales, we first introduce fractional Sobolev spaces on time scales, characterize them, and define weak conformable fractional derivatives. Second, we prove the equivalence of some norms in the introduced spaces and derive their completeness, reflexivity, uniform convexity, and compactness of some imbeddings, which can be regarded as a novelty item. Then, as an application, we present a recent approach via variational methods and critical point theory to obtain the existence of solutions for a $p$-Laplacian conformable fractional differential equation boundary value problem on time scale $\mathbb{T}: T_{\alpha}\left(\left|T_{\alpha}(u)\right|^{p-2} T_{\alpha}(u)\right)(t)=\nabla F(\sigma(t), u(\sigma(t))), \Delta$-a.e. $t \in[a, b]_{\mathbb{K}}^{\kappa^{2}}, u(a)-u(b)=0, T_{\alpha}(u)(a)-T_{\alpha}(u)(b)=0$, where $T_{\alpha}(u)(t)$ denotes the conformable fractional derivative of $u$ of order $\alpha$ at $t, \sigma$ is the forward jump operator, $a, b \in \mathbb{T}, 0<a<b, p>1$, and $F:[0, T]_{\mathbb{T}} \times \mathbb{R}^{N} \rightarrow \mathbb{R}$. By establishing a proper variational setting, we obtain three existence results. Finally, we present two examples to illustrate the feasibility and effectiveness of the existence results.

\section{Introduction}

A time scale $\mathbb{T}$ is an arbitrary nonempty closed subset of the real numbers, which has the topology inherited from the real numbers with the standard topology. In order to unify and generalize continuous and discrete analysis, the calculus on time scales was initiated by Hilger in 1990 (see $[1,2])$. In view of the fact that time scale calculus can be used to model dynamic processes whose time domains are more complicated than the set of integers or real numbers, it plays an important role in various equations and systems arising in economy, biology, ecology, astronomy, and so on (see $[1,3,4])$. During the last decade, there has been a great deal of interest in the study of dynamic equations on time scales and the research in this area is rapidly growing, see [5-10] and the references therein. Nevertheless, these studies are all about integer order dynamic equations on time scales. The existence and multiplicity of solutions for fractional dynamic equations on time scales has received considerably less attention (see $[11,12])$.
It is well known that the fractional calculus refers to differentiation and integration of an arbitrary (noninteger) order. The theory is owed to mathematicians such as Leibniz, Liouville, Riemann, Letnikov, and Grunwald. In this day and age, fractional calculus is one of the most intensively developing areas of mathematical analysis, including several definitions of fractional calculus like RiemannLiouville fractional calculus, Caputo fractional calculus, Grunwald-Letnikov fractional calculus, Hadamard fractional calculus, Riesz fractional calculus, Weyl fractional calculus, Kolwankar-Gangal fractional calculus and so on. Especially, in [13], the authors introduce the conformable fractional calculus on $\mathbb{R}$. In order to unify and generalize the Hilger calculus and the conformable fractional calculus on $\mathbb{R}$, the authors introduce the conformable fractional calculus on time scales and study its properties in [14]. The generation of fractional differential equations is born with the birth of the fractional calculus. Fractional differential equations have gained importance due to their numerous applications in many fields of science and engineering including fluid flow, 
electrical networks, probability and statistics, viscoelasticity, chemical physics and signal processing, and so on, see [15-18] and references therein.

On one hand, there have been many approaches to study solutions of boundary value problems for the fractional differential equations such as lower and upper solution method, monotone iterative method ([19]), fixed-point theorems ([20]), Leray-Schauder theory ([21]), critical point theory ([22]) and so on. But until now, as far as I am concerned, no researchers have applied the critical point theory to study conformable fractional differential equations on time scales. Since it is often very difficult to establish a suitable space and variational functional for conformable fractional differential equations on time scales.

On the other hand, Sobolev spaces are regarded as one of most fundamental tools, especially in the use of variational methods to solve boundary value problems in ordinary and partial differential equations and difference equations, see [23-26]. Sobolev's spaces on a closed interval $[0, T]$ of $\mathbb{R}$ are well known in [24]. For the sake of the study for differential equations on time scales, the authors defined the Sobolev spaces on time scales and studied some of their important properties in [25]. In [26], Jiao and Zhou developed a Caputo fractional derivative space and some of their properties which can be used to study Caputo fractional differential equation boundary value problems via critical point theory.

In view of the above reasons, our main purpose of this paper is to construct fractional Sobolev spaces on time scales via conformable fractional calculus and investigate some of their important properties. As an application, we present a recent approach via variational methods and critical point theory to obtain the existence of solutions for a $p$-Laplacian conformable fractional differential equations boundary value problem on time scale $\mathbb{T}$.

This paper is organized as follows. In Section 2, we introduce the definition of conformable fractional calculus and their important properties. In Section 3, we construct fractional Sobolev spaces on time scales via conformable fractional calculus and investigate some of their important properties. Section 4, as an application of the fractional Sobolev's spaces on time scales, we present a recent approach via variational methods and critical point theory to obtain the existence of solutions for $p$-Laplacian conformable fractional differential equations boundary value problem on time scale $\mathbb{T}$. By making a variational structure on the fractional Sobolev's spaces on time scales, we can reduce the problem of finding solutions of a $p$-Laplacian conformable fractional differential equation boundary value problem on time scale $\mathbb{T}$ to the one of seeking the critical points of a corresponding functional.

\section{Conformable Fractional Calculus on Time Scales and Their Properties}

In this section, we introduce some definitions of conformable fractional derivative and integral and study some of their important properties.
Throughout this paper, we assume $a, b \in \mathbb{T}, 0<a<$ $b$. We start by the definitions of conformable fractional derivative.

For convenience, we denote the intervals $[a, b]_{\mathbb{T}},[a, b)_{\mathbb{T}}$ and $(a, b]_{\mathbb{T}}$ in $\mathbb{T}$ by

$$
\begin{aligned}
& {[a, b]_{\mathbb{T}}=[a, b] \cap \mathbb{T},} \\
& {[a, b)_{\mathbb{T}}=[a, b) \cap \mathbb{T},} \\
& (a, b]_{\mathbb{T}}=(a, b] \cap \mathbb{T},
\end{aligned}
$$

respectively. Note that $[a, b]_{\mathbb{T}}^{\kappa}=[a, b]_{\mathbb{T}}$ if $b$ is left-dense and $[a, b]_{\mathbb{T}}^{\kappa}=[a, b)_{\mathbb{T}}=[a, \rho(b)]_{\mathbb{T}}$ if $b$ is left-scattered. We denote $[a, b]_{\mathbb{T}}^{\kappa^{2}}=\left([a, b]_{\mathbb{T}}^{\kappa}\right)^{\kappa}$, therefore $[a, b]_{\mathbb{I}}^{\kappa^{2}}=[a, b]_{\mathbb{T}}$ if $b$ is leftdense and $[a, b]_{\mathbb{T}}^{\kappa^{2}}=[a, \rho(b)]_{\mathbb{T}}^{\kappa}$ if $b$ is left-scattered.

Let $t \in \mathbb{T}$ and $\delta>0$. We define the $\delta$-neighborhood of $t$ as $\mathscr{V}_{t}:=(t-\delta, t+\delta) \cap \mathbb{T}$. We begin to introduce a new notation: the conformable fractional derivative of order $\alpha \in(0,1]$ for functions defined on arbitrary time scales.

Definition 1 (Definition 1, [14]). Let $f: \mathbb{T} \rightarrow \mathbb{R}, t \in \mathbb{T}^{\kappa}$, and $\alpha \in(0,1]$. For $t>0$, we define $T_{\alpha}(f)(t)$ to be the number (provided it exists) with the property that given any $\epsilon>0$, there is a $\delta$-neighborhood $\mathscr{V}_{t} \subset \mathbb{T}$ of $t, \delta>0$, such that

$$
\begin{aligned}
& \left|[f(\sigma(t))-f(s)] t^{1-\alpha}-T_{\alpha}(f)(t)[\sigma(t)-s]\right| \\
& \quad \leq \epsilon|\sigma(t)-s| \quad \forall s \in \mathscr{V}_{t} .
\end{aligned}
$$

We call $T_{\alpha}(f)(t)$ the conformable fractional derivative of $f$ of order $\alpha$ at $t$, and we define the conformable fractional derivative at 0 as $T_{\alpha}(f)(0)=\lim _{t \rightarrow 0^{+}} T_{\alpha}(f)(t)$.

Definition 2 (Definition 23, [14]). Let $\alpha \in(n, n+1], n \in \mathbb{N}$, and $f: \mathbb{T} \rightarrow \mathbb{R}$ be $n$ times delta differentiable at $t \in \mathbb{T}^{\kappa^{n}}$. We define the conformable fractional derivative of $f$ of order $\alpha$ as $T_{\alpha}(f)(t):=T_{\alpha-n}\left(f^{\Delta^{n}}\right)(t)$.

Definition 3 (Definition 26, [14]). Let $f: \mathbb{T} \rightarrow \mathbb{R}$ be a regulated function. Then the $\alpha$-fractional integral of $f, 0<$ $\alpha \leq 1$, is defined by $\int f(t) \Delta^{\alpha} t:=\int f(t) t^{\alpha-1} \Delta t$.

Definition 4 (Definition 28, [14]). Suppose $f: \mathbb{T} \rightarrow \mathbb{R}$ is a regulated function. Denote the indefinite $\alpha$-fractional integral of $f$ of order $\alpha, \alpha \in(0,1]$, as follows: $F_{\alpha}(t)=$ $\int f(t) \Delta^{\alpha} t$. Then, for all $a, b \in \mathbb{T}$, we define the Cauchy $\alpha$-fractional integral by $\int_{a}^{b} f(t) \Delta^{\alpha} t=F_{\alpha}(b)-F_{\alpha}(a)$.

The $\Delta$-measure $\mu_{\Delta}$ and $\Delta$-integration are defined the same as those in [27].

Definition 5 (Definition 2.3, [28]). Let $B \subset \mathbb{T} . B$ is called $\Delta$ null set if $\mu_{\Delta}(B)=0$. Say that a property $P$ holds $\Delta$-almost everywhere ( $\Delta$-a.e.) on $B$, or for $\Delta$-almost all $(\Delta$-a.a.) $t \in B$ if there is a $\Delta$-null set $E_{0} \subset B$ such that $P$ holds for all $t \in B \backslash E_{0}$.

Definition 6. Assume $f: \mathbb{T} \rightarrow \mathbb{R}$ is a function. Let $A$ is a $\Delta$ measurable subset of $\mathbb{T} . f$ is $\alpha$-integrable on $A$ if and only if $t^{\alpha-1} f$ is integrable on $A$, and $\int_{A} f(t) \Delta^{\alpha} t=\int_{A} f(t) t^{\alpha-1} \Delta t$. 
The conformable fractional calculus of $f: \mathbb{T} \rightarrow \mathbb{R}$ has the following important properties. Before the statement of the properties, we denote

$$
\begin{aligned}
& C_{\mathrm{rd}}\left([a, b]_{\mathbb{T}}, \mathbb{R}\right)=\left\{f:[a, b]_{\mathbb{T}} \longrightarrow \mathbb{R}, f \text { is rd-continuous on }[a,\right. \\
& \left.\quad b]_{\mathbb{T}}\right\}, \\
& C_{0}\left([a, b]_{\mathbb{T}}, \mathbb{R}\right)=\left\{f:[a, b]_{\mathbb{T}} \longrightarrow \mathbb{R}, f \text { is continuous on }[a, b)_{\mathbb{T}}\right. \\
& \left.\quad \text { with compact support in }[a, b)_{\mathbb{T}}\right\}, \\
& C_{\mathrm{rd}}^{\alpha}\left([a, b]_{\mathbb{T}}, \mathbb{R}\right) \\
& \quad=\{f \text { is conformal fractional differentiable of order } \alpha \text { on }[a, \\
& \left.\quad b]_{\mathbb{T}} \text { and } T_{\alpha}(f) \in C_{\mathrm{rd}}\left([a, b]_{\mathbb{T}}, \mathbb{R}\right)\right\}, \\
& C_{0, \mathrm{rd}}^{\alpha}\left([a, b]_{\mathbb{T}}, \mathbb{R}\right)=\left\{f \in C_{\mathrm{rd}}^{\alpha}\left([a, b]_{\mathbb{T}}, \mathbb{R}\right): f(a)=f(b)=0\right\}, \\
& C_{a, b ; \mathrm{rd}}^{\alpha}\left([a, b]_{\mathbb{T}}, \mathbb{R}\right)=\left\{f \in C_{\mathrm{rd}}^{\alpha}\left([a, b]_{\mathbb{T}}, \mathbb{R}\right): f(a)=f(b)\right\} .
\end{aligned}
$$

Lemma 7 (Theorem 4, [14]). Let $\alpha \in(0,1]$. Assume $f: \mathbb{T} \rightarrow$ $\mathbb{R}$ and let $t \in \mathbb{T}^{\kappa}$. The following properties hold:

(i) If $f$ is conformal fractional differentiable of order $\alpha$ at $t>0$, then $f$ is continuous at $t$.

(ii) If $f$ is continuous at $t$ and $t$ is right-scattered, then $f$ is conformable fractional differentiable of order $\alpha$ at $t$ with

$$
T_{\alpha}(f)(t)=f^{\Delta}(t) t^{1-\alpha}
$$

(iii) If $t$ is right-dense, then $f$ is conformable fractional differentiable of order $\alpha$ at $t$ if and only if the limit

$$
\lim _{s \rightarrow t} \frac{f(t)-f(s)}{t-s} t^{1-\alpha}
$$

exists as a finite number. In this case,

$$
T_{\alpha}(f)(t)=f^{\prime}(t) t^{1-\alpha} .
$$

(iv) If $f$ is conformable fractional differentiable of order $\alpha$ at $t$, then

$$
f(\sigma(t))=f(t)+\mu(t) t^{\alpha-1} T_{\alpha}(f)(t) .
$$

Lemma 8 (Theorem 15, [14]). Assume $f, g: \mathbb{T} \rightarrow \mathbb{R}$ are conformable fractional differentiable of order $\alpha$. Then

(i) the sum $f+g: \mathbb{T} \rightarrow \mathbb{R}$ is conformable fractional differentiable with $T_{\alpha}(f+g)=T_{\alpha}(f)+T_{\alpha}(g)$;

(ii) for any $\lambda \in \mathbb{R}, \lambda f: \mathbb{T} \rightarrow \mathbb{R}$ is conformable fractional differentiable with $T_{\alpha}(\lambda f)=\lambda T_{\alpha}(f)$;

(iii) if $f$ and $g$ are continuous, then the product $f g: \mathbb{T} \rightarrow \mathbb{R}$ is conformable fractional differentiable with $T_{\alpha}(f g)=$ $T_{\alpha}(f) g+(f \circ \sigma) T_{\alpha}(g)=T_{\alpha}(f)(g \circ \sigma)+(f) T_{\alpha}(g) ;$

(iv) if $f$ is continuous, then $1 / f$ is conformable fractional differentiable with

$$
T_{\alpha}\left(\frac{1}{f}\right)=-\frac{T_{\alpha}(f)}{f(f \circ \sigma)}
$$

(v) if $f$ and $g$ are continuous, then $f / g$ is conformable fractional differentiable with

$$
T_{\alpha}\left(\frac{f}{g}\right)=\frac{T_{\alpha}(f) g-f T_{\alpha}(g)}{g(g \circ \sigma)} .
$$

Lemma 9 (Theorem 25, [14]). Let $\alpha \in(n, n+1], n \in \mathbb{N}$. The following relation holds:

$$
T_{\alpha}(f)(t)=t^{1+n-\alpha} f^{\Delta^{1+n}}(t)
$$

Remark 10. In (10), when $n=0$, we have $T_{\alpha}(f)(t)=$ $t^{1-\alpha} f^{\Delta}(t), \alpha \in(0,1]$.

Lemma 11 (Theorem 30, [14]). Let $\alpha \in(0,1]$. Then, for any $r d$-continuous function $f: \mathbb{T} \rightarrow \mathbb{R}$, there exists a function $F_{\alpha}: \mathbb{T} \rightarrow \mathbb{R}$ such that $T_{\alpha}\left(F_{\alpha}\right)(t)=f(t)$ for all $t \in \mathbb{T}^{\mathcal{K}}$. Function $F_{\alpha}$ is said to be an $\alpha$-antiderivative of $f$.

Lemma 12 (Theorem 31, [14]). Let $\alpha \in(0,1], a, b, c \in \mathbb{T}, \lambda \in$ $\mathbb{R}$ and $f, g: \mathbb{T} \rightarrow \mathbb{R}$ be two rd-continuous functions. Then,

(i) $\int_{a}^{b}[f(t)+g(t)] \Delta^{\alpha} t=\int_{a}^{b} f(t) \Delta^{\alpha} t+\int_{a}^{b} g(t) \Delta^{\alpha} t$;

(ii) $\int_{a}^{b} \lambda f(t) \Delta^{\alpha} t=\lambda \int_{a}^{b} f(t) \Delta^{\alpha} t$;

(iii) $\int_{a}^{b} f(t) \Delta^{\alpha} t=-\int_{b}^{a} f(t) \Delta^{\alpha} t$;

(iv) $\int_{a}^{b} f(t) \Delta^{\alpha} t=\int_{a}^{c} f(t) \Delta^{\alpha} t+\int_{c}^{b} f(t) \Delta^{\alpha} t$;

(v) $\int_{a}^{a} f(t) \Delta^{\alpha} t=0$;

(vi) if there exist $g: \mathbb{T} \rightarrow \mathbb{R}$ with $|f(t)| \leq g(t)$ for all $t \in[a, b]_{\mathbb{T}}$, then $\left|\int_{a}^{b} f(t) \Delta^{\alpha} t\right| \leq \int_{a}^{b} g(t) \Delta^{\alpha} t ;$

(vii) if $f(t)>0$ for all $t \in[a, b]_{\mathbb{T}}$, then $\int_{a}^{b} f(t) \Delta^{\alpha} t \geq 0$.

Lemma 13 (Theorem 33, [14]). Let $\mathbb{T}$ be a time scale. If $T_{\alpha}(f)(t) \geq 0$ for all $t \in[a, b]_{\mathbb{T}}$, then $f$ is an increasing function on $[a, b]_{\mathbb{T}}$.

Theorem 14. Let $f: \mathbb{T} \rightarrow \mathbb{R}$ be a continuous function on $[a, b]_{\mathbb{T}}$ that is conformal fractional differentiable of order $\alpha$ on $[a, b)_{\mathbb{T}}$ and satisfies $f(a)=f(b)$. Then there exist $\xi, \tau \in[a, b]_{\mathbb{T}}^{\kappa}$ such that $T_{\alpha}(f)(\xi) \leq 0 \leq T_{\alpha}(f)(\tau)$.

Proof. Since the function $f$ is continuous on the compact set $[a, b]_{\mathbb{T}}, f$ assumes its minimum $m$ and its maximum $M$. Therefore there exist $\xi, \tau \in[a, b]_{\mathbb{T}}$ such that $m=f(\xi)$ and $M=f(\tau)$. Since $f(a)=f(b)$, we may assume that $\xi, \tau \in$ $[a, b]_{\mathbb{T}}^{\kappa}$. By Lemma 13, we have $T_{\alpha}(f)(\xi) \leq 0 \leq T_{\alpha}(f)(\tau)$. The proof is complete.

Theorem 15 (mean value theorem). Let $f$ be a continuous function on $[a, b]_{\mathbb{V}}$ which is conformal fractional differentiable of order $\alpha$ on $[a, b]_{\mathbb{T}}^{\kappa}$. Then there exist $\xi, \tau \in[a, b]_{\mathbb{T}}^{\kappa}$ such that

$$
\xi^{\alpha-1} T_{\alpha}(f)(\xi) \leq \frac{f(b)-f(a)}{b-a} \leq \tau^{\alpha-1} T_{\alpha}(f)(\tau) .
$$


Proof. It follows from Lemma 9 that

$$
T_{\alpha}(t)= \begin{cases}t^{1-\alpha}, & 0<\alpha<1 \\ 1, & \alpha=1\end{cases}
$$

Let $h(t)=f(t)-f(a)-((f(b)-f(a)) /(b-a))(t-a)$. Then, the function $h$ is continuous function on $[a, b]_{\mathbb{T}}$ which is conformal fractional differentiable of order $\alpha$ on $(a, b]_{\mathbb{T}}$ and $h(a)=h(b)$. Combining Lemma 8 and (12), we have

$$
\begin{aligned}
& T_{\alpha}(h)(t) \\
& \quad= \begin{cases}T_{\alpha}(f)(t)-\frac{f(b)-f(a)}{b-a}, & \alpha=1 ; \\
T_{\alpha}(f)(t)-\frac{f(b)-f(a)}{b-a} t^{1-\alpha}, & 0<\alpha<1 .\end{cases}
\end{aligned}
$$

Applying Theorem 14 to $h$, there exist $\xi, \tau \in[a, b)_{\mathbb{W}}$ such that $T_{\alpha}(h)(\xi) \leq 0 \leq T_{\alpha}(h)(\tau)$. That is

$$
\xi^{\alpha-1} T_{\alpha}(f)(\xi) \leq \frac{f(b)-f(a)}{b-a} \leq \tau^{\alpha-1} T_{\alpha}(f)(\tau) .
$$

The proof is complete.

Similar to the [Definition 2.9, [26]], we give the following definition of absolutely continuous function.

Definition 16. A function $f:[a, b]_{\mathbb{T}} \rightarrow \mathbb{R}$ is said to be absolutely continuous on $[a, b]_{\mathbb{T}}$ (i.e., $f \in A C\left([a, b]_{\mathbb{T}}, \mathbb{R}\right)$ ), if for every $\epsilon>0$, there exists $\delta>0$ such that if $\left\{\left[a_{k}, b_{k}\right]_{\mathbb{T}}\right\}_{k=1}^{n}$ is a finite pairwise disjoint family of subintervals of $[a, b]_{\mathbb{T}}$ satisfying $\sum_{k=1}^{n}\left(b_{k}-a_{k}\right)<\delta$, then $\sum_{k=1}^{n}\left|f\left(\rho\left(b_{k}\right)\right)-f\left(a_{k}\right)\right|<\epsilon$.

Lemma 17 (Theorem 2.10, [28]). A function $f:[a, b]_{\mathbb{T}} \rightarrow$ $\mathbb{R}$ is absolutely continuous on $[a, b]_{\mathbb{T}}$ if and only if $f$ is delta differentiable $\Delta$-a.e. on $[a, b)_{\mathbb{T}}$ and

$$
f(t)=f(a)+\int_{[a, t]_{\mathbb{T}}} f^{\Delta}(s) \Delta s, \quad \forall t \in[a, b]_{\mathbb{T}} .
$$

Theorem 18. Assume function $f:[a, b]_{\mathbb{T}} \rightarrow \mathbb{R}$ is absolutely continuous on $[a, b]_{\mathbb{T}}$, then $f$ is conformable fractional differentiable of order $\alpha \Delta$-a.e. on $[a, b]_{\mathbb{T}}$ and the following equality is valid:

$$
f(t)=f(a)+\int_{[a, t]_{\mathbb{T}}} T_{\alpha}(f)(s) \Delta^{\alpha} s, \quad \forall t \in[a, b]_{\mathbb{T}} .
$$

Proof. According to Lemma 17, $f$ is delta differentiable $\Delta$-a.e. on $[a, b]_{\mathbb{T}}$. Then, by Remark $10, f$ is conformable fractional differentiable of order $\alpha \Delta$-a.e. on $[a, b]_{\mathbb{T}}$. Therefore, Definition 4 implies that

$$
f(t)=f(a)+\int_{[a, t]_{\mathbb{T}}} T_{\alpha}(f)(s) \Delta^{\alpha} s, \quad \forall t \in[a, b]_{\mathbb{T}} .
$$

The proof is complete.
Lemma 19 (Theorem 2.11, [28]). A function $f, g:[a, b]_{\mathbb{T}} \rightarrow$ $\mathbb{R}$ is absolutely continuous on $[a, b]_{\mathbb{T}}$, then $f g$ is absolutely continuous on $[a, b]_{\mathbb{T}}$ and the following equality is valid:

$$
\begin{gathered}
\int_{[a, b]_{\mathbb{T}}}\left(f^{\Delta} g+f^{\sigma} g^{\Delta}\right)(t) \Delta t=f(b) g(b)-f(a) g(a) \\
=\int_{[a, b]_{\pi}}\left(f g^{\Delta}+f^{\Delta} g^{\sigma}\right)(t) \Delta t .
\end{gathered}
$$

Theorem 20. Assume function $f, g:[a, b]_{\mathbb{T}} \rightarrow \mathbb{R}$ is absolutely continuous on $[a, b]_{\mathbb{T}}$, then $f g$ is absolutely continuous on $[a, b]_{\mathbb{T}}$ and the following equality is valid:

$$
\begin{aligned}
\int_{a}^{b} & \left(T_{\alpha}(f) g+f^{\sigma} T_{\alpha}(g)\right)(t) \Delta^{\alpha} t \\
& =f(b) g(b)-f(a) g(a) \\
& =\int_{a}^{b}\left(f T_{\alpha}(g)+T_{\alpha}(f) g^{\sigma}\right)(t) \Delta^{\alpha} t
\end{aligned}
$$

Proof. The result is obtained by applying Lemmas 8 and 19 and Theorem 18 to the function $f$ and $g$. The proof is complete.

Definition 21 (Definition 2.4, [28]). Let $E \subset \mathbb{T}$ be a $\Delta$ measurable set and let $p \in \overline{\mathbb{R}}=[-\infty,+\infty]$ be such that $p \geq 1$ and let $f: E \rightarrow \overline{\mathbb{R}}$ be a $\Delta$-measurable function. Say that $f$ belongs to $L_{\Delta}^{p}(E, \mathbb{R})$ provided that either

$$
\int_{E}|f(t)|^{p} \Delta t<+\infty \quad \text { if } p \in \mathbb{R}
$$

or there exists a constant $C \in \mathbb{R}$ such that

$$
|f| \leq C \quad \Delta \text {-a.e. on } E \text { if } p=+\infty \text {. }
$$

Definition 22. Let $E \subset \mathbb{T}$ be a $\Delta$-measurable set and let $p \in \overline{\mathbb{R}}=[-\infty,+\infty]$ be such that $p \geq 1$ and let $f: E \rightarrow \overline{\mathbb{R}}$ be a $\Delta$-measurable function. Say that $f$ belongs to $L_{\alpha, \Delta}^{p}(E, \mathbb{R})$ provided that either

$$
\int_{E}|f(t)|^{p} \Delta^{\alpha} t<+\infty \text { if } p \in \mathbb{R}
$$

or there exists a constant $C \in \mathbb{R}$ such that

$$
|f| \leq C \quad \Delta \text {-a.e. on } E \text { if } p=+\infty \text {. }
$$

Lemma 23 (Theorem 2.5, [28]). Let $p \in \overline{\mathbb{R}}$ be such that $p \geq 1$. Then the set $L_{\Delta}^{p}\left([a, b]_{\mathbb{T}}, \mathbb{R}\right)$ is a Banach space together with the norm defined for $f \in L_{\Delta}^{p}\left([a, b]_{\mathbb{T}}, \mathbb{R}\right)$ as

$$
\begin{aligned}
& \|f\|_{L_{\Delta}^{p}\left([a, b]_{\top}, \mathbb{R}\right)} \\
& = \begin{cases}\left(\int_{[a, b]_{\mathbb{T}}}|f(t)|^{p} \Delta t\right)^{1 / p}, & p \in \mathbb{R} ; \\
\inf \left\{C \in \mathbb{R}:|f| \leq C \Delta \text {-a.e. on }[a, b]_{\mathbb{T}}\right\}, & p=+\infty .\end{cases}
\end{aligned}
$$

Moreover, $L_{\Delta}^{2}\left([a, b]_{\mathbb{T}}, \mathbb{R}\right)$ is a Hilbert space together with the inner product given for every $(f, g) \in L_{\Delta}^{p}\left([a, b]_{\mathbb{T}}, \mathbb{R}\right) \times$ $L_{\Delta}^{p}\left([a, b]_{\mathbb{T}}, \mathbb{R}\right) b y$

$$
\langle f, g\rangle_{L_{\Delta}^{2}\left([a, b]_{\mathbb{T}}, \mathbb{R}\right)}=\int_{[a, b]_{\mathbb{T}}} f(t) g(t) \Delta t .
$$


Theorem 24. Let $p \in \overline{\mathbb{R}}$ be such that $p \geq 1$. Then the set $L_{\alpha, \Delta}^{p}\left([a, b]_{\mathbb{T}}, \mathbb{R}\right)(a \geq 0)$ is a Banach space together with the norm defined for $f \in L_{\alpha, \Delta}^{p}\left([a, b]_{\mathbb{T}}, \mathbb{R}\right)$ as

$$
\begin{aligned}
& \|f\|_{L_{\alpha, \Delta}^{p}\left([a, b]_{\mathbb{T}}, \mathbb{R}\right)} \\
& = \begin{cases}\left(\int_{[a, b]_{\mathbb{T}}}|f(t)|^{p} \Delta^{\alpha} t\right)^{1 / p}, & p \in \mathbb{R} ; \\
\inf \left\{C \in \mathbb{R}:|f| \leq C \Delta \text {-a.e. on }[a, b]_{\mathbb{T}}\right\}, & p=+\infty .\end{cases}
\end{aligned}
$$

Moreover, $L_{\alpha, \Delta}^{2}\left([a, b]_{\mathbb{T}}, \mathbb{R}\right)$ is a Hilbert space together with the inner product given for every $(f, g) \in L_{\alpha, \Delta}^{p}\left([a, b]_{\mathbb{T}}, \mathbb{R}\right) \times$ $L_{\alpha, \Delta}^{p}\left([a, b]_{\mathbb{J}}, \mathbb{R}\right) b y$

$$
\langle f, g\rangle_{L_{\alpha, \Delta}^{2}\left([a, b]_{\mathbb{T}}, \mathbb{R}\right)}=\int_{[a, b]_{\mathbb{T}}} f(t) g(t) \Delta^{\alpha} t .
$$

Proof. Let $\left\{u_{n}\right\}_{n \in \mathbb{N}} \subset L_{\alpha, \Delta}^{p}\left([a, b]_{\mathbb{T}}, \mathbb{R}\right)$ be a Cauchy sequence, then we have

$$
\begin{aligned}
& \int_{[a, b]_{\mathbb{T}}}\left|u_{m}(t)-u_{n}(t)\right|^{p} \Delta^{\alpha} t \\
& \quad=\int_{[a, b]_{\mathbb{T}}}\left|u_{m}(t)-u_{n}(t)\right|^{p} t^{\alpha-1} \Delta t \\
& =\int_{[a, b]_{\mathbb{T}}}\left|u_{m}(t) t^{(\alpha-1) / p}-u_{n}(t) t^{(\alpha-1) / p}\right|^{p} \Delta t \longrightarrow 0 \\
& \quad(m, n \rightarrow \infty) .
\end{aligned}
$$

From Lemma 23 and (28), there exists $u_{0} \in L_{\alpha, \Delta}^{p}\left([a, b]_{\mathbb{T}}, \mathbb{R}\right)$ such that

$$
\begin{aligned}
\int_{[a, b]_{\mathbb{T}}}\left|u_{n}(t) t^{(\alpha-1) / p}-u_{0}(t) t^{(\alpha-1) / p}\right|^{p} \Delta t & \longrightarrow 0 \\
& (n \longrightarrow \infty) .
\end{aligned}
$$

Therefore, we can get

$$
\begin{array}{r}
\left\|u_{n}-u_{0}\right\|_{L_{\alpha, \Delta}^{p}\left([a, b]_{\mathbb{T}}, \mathbb{R}\right)}=\int_{[a, b]_{\mathbb{T}}}\left|u_{n}(t)-u_{0}(t)\right|^{p} \Delta^{\alpha} t \\
=\int_{[a, b]_{\mathbb{T}}}\left|u_{n}(t)-u_{0}(t)\right|^{p} t^{\alpha-1} \Delta t \\
=\int_{[a, b]_{\mathbb{T}}}\left|u_{n}(t) t^{(\alpha-1) / p}-u_{0}(t) t^{(\alpha-1) / p}\right|^{p} \Delta t \longrightarrow 0 \\
(n \longrightarrow \infty) .
\end{array}
$$

Thus, the space $L_{\alpha, \Delta}^{p}\left([a, b]_{\mathbb{T}}, \mathbb{R}\right)$ is a Banach space together with the norm $\|\cdot\|_{L_{\alpha, \Delta}^{p}\left([a, b]_{\mathbb{T}}, \mathbb{R}\right)}$.

Clearly, $L_{\alpha, \Delta}^{2}\left([a, b]_{\mathbb{V}}, \mathbb{R}\right)$ is a Hilbert space together with the inner product given for every $(f, g) \in L_{\alpha, \Delta}^{p}\left([a, b]_{\mathbb{T}}, \mathbb{R}\right) \times$ $L_{\alpha, \Delta}^{p}\left([a, b]_{\mathbb{T}}, \mathbb{R}\right)$ by

$$
\langle f, g\rangle_{L_{\alpha, \Delta}^{2}\left([a, b]_{\mathbb{T}}, \mathbb{R}\right)}=\int_{[a, b]_{\mathbb{T}}} f(t) g(t) \Delta^{\alpha} t .
$$

Lemma 25 (Proposition 2.6, [28]). Suppose $p \in \mathbb{\mathbb { R }}$ and $p \geq 1$. Let $p^{\prime} \in \mathbb{\mathbb { R }}$ be such that $1 / p+1 / p^{\prime}=1$. Then, if $f \in L_{\Delta}^{p}\left([a, b]_{\mathbb{T}}, \mathbb{R}\right)$ and $g \in L_{\Delta}^{p^{\prime}}\left([a, b]_{\mathbb{T}}, \mathbb{R}\right)$, then $f \cdot g \in$ $L_{\Delta}^{1}\left([a, b]_{\mathbb{T}}, \mathbb{R}\right)$ and

$$
\|f \cdot g\|_{L_{\Delta}^{1}\left([a, b]_{\mathbb{T}}, \mathbb{R}\right)} \leq\|f\|_{L_{\Delta}^{p}\left([a, b]_{\mathbb{T}}, \mathbb{R}\right)} \cdot\|g\|_{L_{\Delta}^{p^{\prime}}\left([a, b]_{\mathbb{T}} ; \mathbb{R}\right)} \cdot
$$

Theorem 26. Suppose $p \in \overline{\mathbb{R}}$ and $p \geq 1$. Let $p^{\prime} \in \overline{\mathbb{R}}$ be such that $1 / p+1 / p^{\prime}=1$. Then, if $f \in L_{\alpha, \Delta}^{p}\left([a, b]_{\mathbb{T}}, \mathbb{R}\right)$ and $g \in$ $L_{\alpha, \Delta}^{p^{\prime}}\left([a, b]_{\mathbb{T}}, \mathbb{R}\right)$, then $f \cdot g \in L_{\alpha, \Delta}^{1}\left([a, b]_{\mathbb{T}}, \mathbb{R}\right)$ and

$$
\|f \cdot g\|_{L_{\alpha, \Delta}^{1}\left([a, b]_{\mathbb{T}}, \mathbb{R}\right)} \leq\|f\|_{L_{\alpha, \Delta}^{p}\left([a, b]_{\mathbb{T}}, \mathbb{R}\right)} \cdot\|g\|_{L_{\alpha, \Delta}^{p^{\prime}\left([a, b]_{\mathbb{T}} ; \mathbb{R}\right)}} \cdot
$$

Proof. By Lemma 25, we assert

$$
\begin{aligned}
\| f & \cdot g \|_{L_{\alpha, \Delta}^{1}\left([a, b]_{\mathbb{T}}, \mathbb{R}\right)}=\int_{[a, b]_{\mathbb{T}}}|f g| \Delta^{\alpha} t \\
& =\int_{[a, b]_{\mathbb{T}}}|f g| t^{\alpha-1} \Delta t \\
& =\int_{[a, b]_{\mathbb{T}}}\left(|f| t^{(\alpha-1) / p}\right)\left(|g| t^{(\alpha-1) / p^{\prime}}\right) \Delta t \\
& \leq\left(\int_{[a, b]_{\mathbb{T}}}\left(|f| t^{(\alpha-1) / p}\right)^{p} \Delta t\right)^{1 / p} \\
& \cdot\left(\int_{[a, b]_{\mathbb{T}}}\left(|g| t^{(\alpha-1) / p^{\prime}}\right)^{p} \Delta t\right)^{1 / p^{\prime}} \\
& =\left(\int_{[a, b]_{\mathbb{T}}}|f|^{p} t^{\alpha-1} \Delta t\right)^{1 / p}\left(\int_{[a, b]_{\mathbb{T}}}|g|^{p^{\prime}} t^{\alpha-1} \Delta t\right)^{1 / p^{\prime}} \\
& =\|f\|_{L_{\alpha, \Delta}^{p}\left([a, b]_{\mathbb{T}}, \mathbb{R}\right)} \cdot\|g\|_{L_{\alpha, \Delta}^{p^{\prime}}\left([a, b]_{\mathbb{T}} ; \mathbb{R}\right)} \cdot
\end{aligned}
$$

The proof is complete.

Lemma 27 (Proposition 2.7, [28]). If $p \in \mathbb{R}$ and $p \geq 1$, then, the set $C_{0}\left([a, b]_{\mathbb{T}}, \mathbb{R}\right)$ is dense in $L_{\Delta}^{p}\left([a, b]_{\mathbb{T}}, \mathbb{R}\right)$.

Theorem 28. Let $f \in L_{\alpha, \Delta}^{1}\left([a, b]_{\mathbb{T}}, \mathbb{R}\right)$ be such that the following equality is true:

$$
\int_{[a, b]_{\mathbb{T}}} f(t) u(t) \Delta^{\alpha} t=0, \quad \forall u \in C_{0}\left([a, b]_{\mathbb{T}}, \mathbb{R}\right)
$$

then

$$
f \equiv 0, \quad \Delta \text {-a.e. on }[a, b]_{\mathbb{T}} \text {. }
$$

Proof. For every $\epsilon>0$, the density of $C_{0}\left([a, b]_{\mathbb{T}}, \mathbb{R}\right)$ in $L_{\Delta}^{p}\left([a, b]_{\mathbb{T}}, \mathbb{R}\right)$ guarantees the existence of $f_{1} \in C_{0}\left([a, b]_{\mathbb{T}}, \mathbb{R}\right)$ such that

$$
\int_{[a, b]_{\mathbb{T}}}\left|f_{1}(t)-f(t)\right| \Delta^{\alpha} t<\epsilon
$$


and so, by Lemma 12, (35) and (37), we deduce that for every $u \in C_{0}\left([a, b]_{\mathbb{T}}, \mathbb{R}\right)$, it is true that

$$
\begin{aligned}
& \left|\int_{[a, b]_{\mathbb{T}}} f(t) u(t) \Delta^{\alpha} t\right| \\
& \quad \leq\|u\|_{C\left([a, b]_{\mathbb{T}}, \mathbb{R}\right)} \int_{[a, b]_{\mathbb{T}}}\left|f_{1}(t)-f(t)\right| t^{\alpha-1} \Delta t \\
& \quad=\epsilon a^{\alpha-1}\|u\|_{C\left([a, b]_{\mathbb{T}}, \mathbb{R}\right)} .
\end{aligned}
$$

Because the sets

$$
\begin{aligned}
& A_{1}=\left\{t \in[a, b) \mid f_{1}(t) \geq \epsilon\right\}, \\
& A_{2}=\left\{t \in[a, b) \mid f_{1}(t) \leq-\epsilon\right\}
\end{aligned}
$$

are compact and disjoint subsets of $[a, b]_{\mathbb{T}}$, Urysohn's lemma allows constructing a function $u_{0}:[a, b]_{\mathbb{T}} \rightarrow \mathbb{R}$ which belongs to $C_{0}\left([a, b]_{\mathbb{V}}, \mathbb{R}\right)$ and it verifies

$$
\begin{gathered}
u_{0}(t)= \begin{cases}1, & t \in A_{1}, \\
-1, & t \in A_{2},\end{cases} \\
|u(t)| \leq 1 \quad \text { on }[a, b]_{\mathbb{T}}
\end{gathered}
$$

so that, by defining $A=A_{1} \cup A_{2}$, Theorem 15, (38), (39), and (40), we have that

$$
\begin{aligned}
\int_{[a, b]_{\top}}\left|f_{1}(t)\right| \Delta^{\alpha} t= & \int_{[a, b]_{\top}} f_{1}(t) u_{0}(t) \Delta^{\alpha} t \\
& -\int_{[a, b]_{\top} \backslash A} f_{1}(t) u_{0}(t) \Delta^{\alpha} t \\
& +\int_{[a, b]_{\top} \backslash A}\left|f_{1}(t)\right| \Delta^{\alpha} t \\
\leq & \epsilon a^{\alpha-1}+2 \epsilon a^{\alpha-1}(b-a) .
\end{aligned}
$$

As a consequence of the arbitrary choice of $\epsilon>0$, by (41), we achieve (36). The proof is complete.

Theorem 29. Let $f \in L_{\alpha, \Delta}^{1}\left([a, b]_{\mathbb{T}}, \mathbb{R}\right)$. Then, a necessary and sufficient condition for the validity of the equality

$$
\int_{a}^{b} f(t) T_{\alpha}(h)(t) \Delta^{\alpha} t=0, \quad \forall h \in C_{0, \mathrm{rd}}^{\alpha}\left([a, b]_{\mathbb{T}}, \mathbb{R}\right)
$$

is the existence of a constant $C \in \mathbb{R}$ such that

$$
f \equiv C \quad \Delta \text {-a.e. on }[a, b]_{\mathbb{T}} .
$$

Proof. If $f \equiv C \Delta$-a.e. on $[a, b]_{\mathbb{T}}$, for any $h \in C_{0, \mathrm{rd}}^{\alpha}\left([a, b]_{\mathbb{T}}, \mathbb{R}\right)$, from Lemma 12 and the definition of $C_{0, \mathrm{rd}}^{\alpha}\left([a, b]_{\mathbb{T}}, \mathbb{R}\right)$, one has

$$
\begin{aligned}
& \int_{a}^{b} f(t) T_{\alpha}(h)(t) \Delta^{\alpha} t=C \int_{a}^{b} T_{\alpha}(h)(t) \Delta^{\alpha} t \\
& =C(h(b)-h(a))=0 .
\end{aligned}
$$

Conversely, take $u \in C_{0}\left([a, b]_{\mathbb{T}}, \mathbb{R}\right)$, by defining $h, g$ : $[a, b]_{\mathbb{T}} \rightarrow \mathbb{R}$ as

$$
\begin{aligned}
& h(t)= \begin{cases}u(t)-\frac{\int_{[a, b]_{\mathbb{T}}} u(s) \Delta^{\alpha} s}{\int_{[a, b]_{\mathbb{T}}} 1 \Delta^{\alpha} s}, & t \in[a, b]_{\mathbb{T}}, \\
-\frac{\int_{[a, b]_{\mathbb{T}}} u(s) \Delta^{\alpha} s}{\int_{[a, b]_{\mathbb{T}}} 1 \Delta^{\alpha} s}, & t=b, \\
g(t)=\int_{[a, b]_{\mathbb{T}}} u(s) \Delta^{\alpha} s,\end{cases}
\end{aligned}
$$

the fundamental theorem of conformable fractional calculus establishes that $g \in C_{0, \mathrm{rd}}^{\alpha}\left([a, b]_{\mathbb{T}}, \mathbb{R}\right)$ and so equality (42) yields

$$
\begin{aligned}
0 & =\int_{[a, b]_{\mathbb{T}}} f(t)\left(u(t)-\frac{\int_{[a, b]_{\mathbb{T}}} u(s) \Delta^{\alpha} s}{\int_{[a, b]_{\mathbb{T}}} 1 \Delta^{\alpha} s}\right) \Delta^{\alpha} t \\
& =\int_{[a, b]_{\mathbb{T}}}\left(f(t)-\frac{\int_{[a, b]_{\mathbb{T}}} f(s) \Delta^{\alpha} s}{\int_{[a, b]_{\mathbb{T}}} 1 \Delta^{\alpha} s}\right) u(t) \Delta^{\alpha} t .
\end{aligned}
$$

Thereby, Theorem 28 and (46) allow to deduce (43) with $C=$ $\int_{[a, b]_{\mathbb{T}}} f(s) \Delta^{\alpha} s / \int_{[a, b]_{\mathbb{T}}} 1 \Delta^{\alpha} s$. The proof is complete.

Next, we introduce the conformable fractional calculus on time scales for vector-valued functions and study some of their important properties.

Definition 30. Assume $f: \mathbb{T} \rightarrow \mathbb{R}^{N}$ is a function, $f(t)=$ $\left(f^{1}(t), f^{2}(t), \ldots, f^{N}(t)\right)$ and let $t \in \mathbb{T}^{\mathcal{K}}$. Then one defines $T_{\alpha}(f)(t)=\left(T_{\alpha}\left(f^{1}\right)(t), T_{\alpha}\left(f^{2}\right)(t), \ldots, T_{\alpha}\left(f^{N}\right)(t)\right)$ (provided it exists). One calls $T_{\alpha}(f)(t)$ the conformable fractional derivative of $f$ of order $\alpha$ at $t$. The function $f$ is conformal fractional differentiable of order $\alpha$ provided $T_{\alpha}(f)$ exists for all $t \in \mathbb{T}^{\kappa}$. The function $T_{\alpha}(f): \mathbb{T}^{\kappa} \rightarrow \mathbb{R}^{N}$ is then called the conformable fractional derivative of $f$ of order $\alpha$.

Definition 31. Let $\mathbb{T}$ be a time scale, $\alpha \in(n, n+1], n \in \mathbb{N}$, and let $f: \mathbb{T} \rightarrow \mathbb{R}^{N}$ be $n$ times delta differentiable at $t \in \mathbb{T}^{\kappa^{n}}$. We define the conformable fractional derivative of $f$ of order $\alpha$ as $T_{\alpha}(f)(t):=T_{\alpha-n}\left(f^{\Delta^{n}}\right)(t)$.

Definition 32. Assume $f: \mathbb{T} \rightarrow \mathbb{R}^{N}$ is a function and $f(t)=\left(f^{1}(t), f^{2}(t), \ldots, f^{N}(t)\right)$. Let $A$ be a $\Delta$-measurable subset of $\mathbb{T}$. Then $f$ is $\alpha$-integrable on $A$ if and only if $f^{i}(i=1,2, \ldots, N)$ are $\alpha$-integrable on $A$, and $\int_{A} f(t) \Delta^{\alpha} t=$ $\left(\int_{A} f^{1}(t) \Delta^{\alpha} t, \int_{A} f^{2}(t) \Delta^{\alpha} t, \ldots, \int_{A} f^{N}(t) \Delta^{\alpha} t\right)$.

From Definitions 30 and 32, we have the following theorems.

Theorem 33. Let $\alpha \in(0,1]$. Assume $f: \mathbb{T} \rightarrow \mathbb{R}^{N}$ and let $t \in \mathbb{T}^{\kappa}$. The following properties hold:

(i) If $f$ is conformal fractional differentiable of order $\alpha$ at $t>0$, then $f$ is continuous at $t$. 
(ii) If $f$ is continuous at $t$ and $t$ is right-scattered, then $f$ is conformable fractional differentiable of order $\alpha$ at $t$ with

$$
T_{\alpha}(f)(t)=\frac{1}{\mu(t)}[f(\sigma(t))-f(t)] t^{1-\alpha} .
$$

(iii) If $t$ is right-dense, then $f$ is conformable fractional differentiable of order $\alpha$ at $t$ if and only if the limit

$$
\lim _{s \rightarrow t} \frac{1}{t-s}[f(t)-f(s)] t^{1-\alpha}
$$

exists as a finite number. In this case,

$$
T_{\alpha}(f)(t)=\lim _{s \rightarrow t} \frac{1}{t-s}[f(t)-f(s)] t^{1-\alpha} .
$$

(iv) If $f$ is conformable fractional differentiable of order $\alpha$ at $t$, then

$$
f(\sigma(t))=f(t)+\mu(t) t^{\alpha-1} T_{\alpha}(f)(t) .
$$

Theorem 34. Assume $f, g: \mathbb{T} \rightarrow \mathbb{R}^{N}$ are conformable fractional differentiable of order $\alpha$. Then,

(i) the sum $f+g: \mathbb{T} \rightarrow \mathbb{R}^{N}$ is conformable fractional differentiable with $T_{\alpha}(f+g)=T_{\alpha}(f)+T_{\alpha}(g)$;

(ii) for any $\lambda \in \mathbb{R}, \lambda f: \mathbb{T} \rightarrow \mathbb{R}^{N}$ is conformable fractional differentiable with $T_{\alpha}(\lambda f)=\lambda T_{\alpha}(f)$;

(iii) if $f$ and $g$ are continuous, then the product $f g$ : $\mathbb{T} \rightarrow \mathbb{R}^{N}$ is conformable fractional differentiable with $T_{\alpha}(f g)=T_{\alpha}(f) g+(f \circ \sigma) T_{\alpha}(g)=T_{\alpha}(f)(g \circ \sigma)+$ ( $f) T_{\alpha}(g)$.

Theorem 35. Let $\alpha \in(0,1], a, b, c \in \mathbb{T}, \lambda \in \mathbb{R}$ and $f, g$ : $\mathbb{T} \rightarrow \mathbb{R}^{N}$ be two rd-continuous functions. Then,

(i) $\int_{a}^{b}[f(t)+g(t)] \Delta^{\alpha} t=\int_{a}^{b} f(t) \Delta^{\alpha} t+\int_{a}^{b} g(t) \Delta^{\alpha} t$;

(ii) $\int_{a}^{b} \lambda f(t) \Delta^{\alpha} t=\lambda \int_{a}^{b} f(t) \Delta^{\alpha} t$;

(iii) $\int_{a}^{b} f(t) \Delta^{\alpha} t=-\int_{b}^{a} f(t) \Delta^{\alpha} t$;

(iv) $\int_{a}^{b} f(t) \Delta^{\alpha} t=\int_{a}^{c} f(t) \Delta^{\alpha} t+\int_{c}^{b} f(t) \Delta^{\alpha} t$;

(v) $\int_{a}^{a} f(t) \Delta^{\alpha} t=0$;

(vi) if there exist $g: \mathbb{T} \rightarrow \mathbb{R}$ with $|f(t)| \leq|g(t)|$ for all $t \in[a, b]_{\mathbb{T}}$, then $\left|\int_{a}^{b} f(t) \Delta^{\alpha} t\right| \leq \int_{a}^{b}|g(t)| \Delta^{\alpha} t$.

Definition 36 (Definition 2.6, [25]). A function $f: \mathbb{T} \rightarrow \mathbb{R}^{N}$ is called rd-continuous provided it is continuous at rightdense points in $\mathbb{T}$ and its left-sided limits exist (finite) at leftdense points in $\mathbb{T}$.

As we know from general theory of Sobolev's spaces, another important class of functions is just the absolutely continuous functions on time scales. Similar to Definition 2.11, [23], we give the following definition of absolutely continuous function.
Definition 37. A function $f:[a, b]_{\mathbb{T}} \rightarrow \mathbb{R}^{N}, f(t)=$ $\left(f^{1}(t), f^{2}(t), \ldots, f^{N}(t)\right)$. We say $f$ is absolutely continuous on $[a, b]_{\mathbb{T}}$ (i.e. $f \in A C\left([a, b]_{\mathbb{T}}, \mathbb{R}^{N}\right)$ ), if for every $\epsilon>0$, there exist $\delta>0$ such that if $\left\{\left[a_{k}, b_{k}\right]_{\mathbb{T}}\right\}_{k=1}^{n}$ is a finite pairwise disjoint family of subintervals of $[a, b]_{\mathbb{T}}$ satisfying $\sum_{k=1}^{n}\left(b_{k}-a_{k}\right)<\delta$, then $\sum_{k=1}^{n}\left|f\left(\rho\left(b_{k}\right)\right)-f\left(a_{k}\right)\right|<\epsilon$.

Remark 38 (Remark 2.1, [25]). By Definitions 22 and 30, we have that $f \in A C\left([a, b]_{\mathbb{T}}, \mathbb{R}^{N}\right)$ if and only if $f^{i} \in$ $A C\left([a, b]_{\mathbb{T}}, \mathbb{R}\right)(i=1,2, \ldots, N)$.

Absolutely continuous functions have the following properties.

Combining Definitions 30, 36, Theorems 18 and 20, we have the following theorems.

Theorem 39. Assume function $f:[a, b]_{\mathbb{T}} \rightarrow \mathbb{R}^{N}$ is absolutely continuous on $[a, b]_{\mathbb{T}}$, then $f$ is conformable fractional differentiable of order $\alpha \Delta$-a.e. on $[a, b]_{\mathbb{T}}$ and the following equality is valid:

$$
f(t)=f(a)+\int_{[a, b]_{\mathbb{T}}} T_{\alpha}(f)(s) \Delta^{\alpha} s, \quad \forall t \in[a, b]_{\mathbb{T}} .
$$

Theorem 40. A function $f, g:[a, b]_{\mathbb{T}} \rightarrow \mathbb{R}^{N}$ is absolutely continuous on $[a, b]_{\mathbb{T}}$, then $f g$ is absolutely continuous on $[a, b]_{\mathbb{T}}$ and the following equality is valid:

$$
\begin{aligned}
& \int_{[a, b]_{\mathbb{T}}}\left(\left(T_{\alpha} f(t), g(t)\right)+\left(f^{\sigma}(t), T_{\alpha} g(t)\right)\right) \Delta^{\alpha} t \\
& =(f(b), g(b))-(f(a), g(a)) \\
& =\int_{[a, b]_{\mathbb{T}}}\left(\left(f(t), T_{\alpha}(g)(t)\right)\right. \\
& \left.+\left(T_{\alpha}(f)(t), g^{\sigma}(t)\right)\right) \Delta^{\alpha} t .
\end{aligned}
$$

\section{Conformable Fractional Sobolev's Spaces on Time Scales and Their Properties}

In this section, we develop the fractional Sobolev's spaces on time scales via conformable fractional calculus and their important properties.

For $p \in \mathbb{R}, p \geq 1$, we set the space

$$
\begin{aligned}
& L_{\alpha, \Delta}^{p}\left([a, b]_{\mathbb{T}}, \mathbb{R}^{N}\right) \\
& \quad=\left\{u:[a, b]_{\mathbb{T}} \longrightarrow \mathbb{R}^{N}: \int_{[a, b]_{\mathbb{T}}}|f(t)|^{p} \Delta^{\alpha} t<+\infty\right\}
\end{aligned}
$$

with the norm

$$
\|f\|_{L_{\alpha, \Delta}^{p}}=\left(\int_{[a, b]_{\mathbb{T}}}|f(t)|^{p} \Delta^{\alpha} t\right)^{1 / p}
$$

We can obtain the following theorem. 
Theorem 41. Assume $p \geq 1$ is a real number. Then the space $L_{\alpha, \Delta}^{p}\left([a, b]_{\mathbb{T}}, \mathbb{R}^{N}\right)$ is a Banach space together with the norm $\|\cdot\|_{L_{\alpha \Delta}^{p}}$. Especially, when $p=2, L_{\alpha, \Delta}^{2}\left([a, b]_{\mathbb{T}}, \mathbb{R}^{N}\right)$ is a Hilbert space together with the inner product given for every $(f, g) \in$ $L_{\alpha, \Delta}^{p}\left([a, b)_{\mathbb{T}^{N}}, \mathbb{R}^{N}\right) \times L_{\alpha, \Delta}^{p}\left([a, b]_{\mathbb{T}}, \mathbb{R}^{N}\right)$ by

$$
\langle f, g\rangle_{L_{\alpha, \Delta}^{2}}=\int_{[a, b]_{\top}}(f(t), g(t)) \Delta^{\alpha} t
$$

where $(\cdot, \cdot)$ denotes the inner product in $\mathbb{R}^{N}$.

Proof. Let $\left\{u_{n}\right\}_{n \in \mathbb{N}} \subset L_{\alpha, \Delta}^{p}\left([a, b]_{\mathbb{T}}, \mathbb{R}^{N}\right)$ be a Cauchy sequence, $u_{n}(t)=\left(u_{n}^{1}(t), u_{n}^{2}(t), \ldots, u_{n}^{N}(t)\right)$. Hence, one has

$$
\begin{array}{r}
\left\|u_{m}-u_{n}\right\|_{L_{\alpha, \Delta}^{p}}=\left(\int_{[a, b]_{\top}}\left|u_{n}(t)-u_{m}(t)\right|^{p} \Delta^{\alpha} t\right)^{1 / p} \\
=\left(\int_{[a, b]_{\top}}\left(\sum_{i=1}^{N}\left|u_{n}^{i}(t)-u_{m}^{i}(t)\right|^{2}\right)^{p / 2} \Delta^{\alpha} t\right)^{1 / p} \longrightarrow 0 \\
(m, n \longrightarrow \infty) .
\end{array}
$$

It follows from (56) that

$$
\begin{aligned}
& \left\|u_{m}^{i}-u_{n}^{i}\right\|_{L_{\alpha, \Delta}^{p}\left([a, b]_{\top}, \mathbb{R}\right)} \\
& \quad=\left(\int_{[a, b]_{\top}}\left|u_{n}^{i}(t)-u_{m}^{i}(t)\right|^{p} \Delta^{\alpha} t\right)^{1 / p} \longrightarrow 0 \\
& \quad(m, n \longrightarrow \infty)
\end{aligned}
$$

for any $i \in\{1,2, \ldots, N\}$. Therefore, $\left\{u_{n}^{i}\right\}_{n \in \mathbb{N}}(i=1,2, \ldots, N)$ are the Cauchy sequences of $L_{\alpha, \Delta}^{p}\left([a, b]_{\mathbb{T}}, \mathbb{R}\right)$. By Theorem 24 , there exist $\bar{u}^{i} \in L_{\alpha, \Delta}^{p}\left([a, b]_{\mathbb{T}}, \mathbb{R}\right)(i=1,2, \ldots, N)$ such that

$$
\left\|u_{n}^{i}-\bar{u}^{i}\right\|_{L_{\Delta}^{p}\left([a, b]_{T}, \mathbb{R}\right)} \longrightarrow 0 \quad(n \longrightarrow \infty) .
$$

We let $\bar{u}(t)=\left(\bar{u}^{1}(t), \bar{u}^{2}(t), \ldots, \bar{u}^{N}(t)\right)$. On one hand, we can get

$$
\begin{aligned}
\int_{[a, b]_{\top}}|\bar{u}|^{p} \Delta^{\alpha} t & =\int_{[a, b]_{\pi}}\left(\sum_{i=1}^{N}\left|\bar{u}^{i}\right|^{2}\right)^{p / 2} \Delta^{\alpha} t \\
& \leq N^{p / 2} \int_{[a, b]_{\top}} \sum_{i=1}^{N}\left|\bar{u}^{i}\right|^{p} \Delta^{\alpha} t \\
& =N^{p / 2} \sum_{i=1}^{N} \int_{[a, b]_{\pi}}\left|\bar{u}^{i}\right|^{p} \Delta^{\alpha} t<+\infty .
\end{aligned}
$$

Thus, $\bar{u} \in L_{\Delta}^{p}\left([a, b]_{\mathbb{T}}, \mathbb{R}^{N}\right)$. On the other hand, according to (58), we have

$$
\begin{aligned}
& \int_{[a, b]_{\top}}\left|u_{n}(t)-\bar{u}(t)\right|^{p} \Delta^{\alpha} t \\
& =\int_{[a, b]_{\top}}\left(\sum_{i=1}^{N}\left|u_{n}^{i}(t)-\bar{u}^{i}(t)\right|^{2}\right)^{p / 2} \\
& \leq N^{p / 2} \int_{[a, b]_{\top}} \sum_{i=1}^{N}\left|u_{n}^{i}(t)-\bar{u}^{i}(t)\right|^{p} \Delta^{\alpha} t \\
& =N^{p / 2} \sum_{i=1}^{N} \int_{[a, b]_{\top}}\left|u_{n}^{i}(t)-\bar{u}^{i}(t)\right|^{p} \Delta^{\alpha} t \\
& =N^{p / 2} \sum_{i=1}^{N}\left\|u_{n}^{i}-\bar{u}^{i}\right\|_{L_{\alpha, \Delta}^{p}\left([a, b]_{T}, \mathbb{R}\right)} \longrightarrow 0
\end{aligned}
$$

$$
(n \longrightarrow \infty)
$$

From (60), $u_{n} \rightarrow \bar{u}$ in $L_{\alpha, \Delta}^{p}\left([a, b]_{\mathbb{V}}, \mathbb{R}^{N}\right)$; therefore, the space $L_{\alpha, \Delta}^{p}\left([a, b]_{\mathbb{T}}, \mathbb{R}^{n}\right)$ is a Banach space together with the norm $\|\cdot\|_{L_{\alpha, \Delta}^{p}}$.

Evidently, $L_{\alpha, \Delta}^{2}\left([a, b]_{\pi}, \mathbb{R}^{N}\right)$ is a Hilbert space together with the inner product given for every $(f, g) \in L_{\alpha, \Delta}^{p}\left([a, b)_{\mathbb{T}^{N}}\right.$, $\left.\mathbb{R}^{N}\right) \times L_{\alpha, \Delta}^{p}\left([a, b]_{\mathbb{T}} ; \mathbb{R}^{N}\right)$ by

$$
\langle f, g\rangle_{L_{\alpha, \Delta}^{2}}=\int_{[a, b]_{\top}}(f(t), g(t)) \Delta^{\alpha} t .
$$

The proof is complete.

Now, we introduce the conformable fractional Sobolev's spaces on $[a, b]_{\mathrm{T}}$. For the sake of convenience, in the sequel, we will let $u^{\sigma}(t)=u(\sigma(t))$ and

$$
\begin{aligned}
& C_{\mathrm{rd}}\left([a, b]_{\mathbb{T}}, \mathbb{R}^{N}\right)=\left\{f:[a, b]_{\mathbb{T}} \longrightarrow \mathbb{R}^{N}, f \text { is rd-continuous on }[a, b]_{\mathbb{T}}\right\}, \\
& C_{\mathrm{rd}}^{\alpha}\left([a, b]_{\mathbb{T}}, \mathbb{R}^{N}\right) \\
& \quad=\left\{f \text { is conformal fractional differentiable of order } \alpha \text { on }[a, b]_{\mathbb{T}} \text { and } T_{\alpha}(f) \in C_{\mathrm{rd}}\left([a, b]_{\mathbb{T}}, \mathbb{R}^{N}\right)\right\}, \\
& C_{a, b ; \mathrm{rd}}^{\alpha}\left([a, b]_{\mathbb{T}}, \mathbb{R}^{N}\right)=\left\{f \in C_{\mathrm{rd}}^{\alpha}\left([a, b]_{\mathbb{T}}, \mathbb{R}^{N}\right): f(a)=f(b)\right\} .
\end{aligned}
$$


Definition 42. Let $p \in \mathbb{R}$ be such that $p \geq 1$ and $u:[a, b]_{\mathbb{T}} \rightarrow$ $\mathbb{R}^{N}$. One says that $u \in W_{\Delta ; a, b}^{\alpha, p}\left([a, b]_{\mathbb{T}}, \mathbb{R}^{N}\right)$ if and only if $u \in$ $L_{\alpha, \Delta}^{p}\left([a, b]_{\mathbb{T}}, \mathbb{R}^{N}\right), T_{\alpha} u \in L_{\alpha, \Delta}^{p}\left([a, b]_{\mathbb{T}}, \mathbb{R}^{N}\right)$, and there exists $g:[a, b]_{\mathbb{T}}^{\kappa} \rightarrow \mathbb{R}^{N}$ such that $g \in L_{\alpha, \Delta}^{p}\left([a, b]_{\mathbb{T}}, \mathbb{R}^{N}\right)$ and

$$
\begin{aligned}
& \int_{[a, b]_{\mathbb{T}}}\left(u(t), T_{\alpha}(\phi)(t)\right) \Delta^{\alpha} t \\
&=-\int_{[a, b]_{\mathbb{T}}}\left(g(t), \phi^{\sigma}(t)\right) \Delta^{\alpha} t, \\
& \forall \phi \in C_{a, b ; \mathrm{rd}}^{\alpha}\left([a, b]_{\mathbb{T}}, \mathbb{R}^{N}\right) .
\end{aligned}
$$

For $p \in \mathbb{R}, p \geq 1$, we denote

$$
\begin{aligned}
& V_{\Delta ; a, b}^{\alpha, p}\left([a, b]_{\mathbb{T}}, \mathbb{R}^{N}\right)=\left\{u \in A C\left([a, b]_{\mathbb{T}}, \mathbb{R}^{N}\right): T_{\alpha}(u)\right. \\
& \left.\quad \in L_{\alpha, \Delta}^{p}\left([a, b]_{\mathbb{V}}, \mathbb{R}^{N}\right), u(a)=u(b)\right\} .
\end{aligned}
$$

Remark 43. Theorems 39 and 40 imply that for every $p \in \mathbb{R}$ with $p \geq 1$,

$$
V_{\Delta ; a, b}^{\alpha, p}\left([a, b]_{\mathbb{T}}, \mathbb{R}^{N}\right) \subset W_{\Delta ; a, b}^{\alpha, p}\left([a, b]_{\mathbb{T}}, \mathbb{R}^{N}\right) .
$$

Next, we will point out that both sets are, as class of functions, equivalent. For the sake of this purpose, we prove the following theorems firstly.

Theorem 44. Suppose $f \in L_{\alpha, \Delta}^{1}\left([a, b]_{\mathbb{T}}, \mathbb{R}^{N}\right)$. Then, a necessary and sufficient condition for the validity of the equality

$$
\begin{aligned}
& \int_{[a, b]_{\mathbb{T}}}\left(f(t), T_{\alpha}(h)(t)\right) \Delta^{\alpha} t=0, \\
& \forall h \in C_{a, b ; \mathrm{rd}}^{\alpha}\left([a, b]_{\mathbb{T}}, \mathbb{R}^{N}\right)
\end{aligned}
$$

is the existence of a constant $C \in \mathbb{R}^{N}$ such that

$$
f \equiv C \quad \Delta \text {-a.e. on }[a, b]_{\mathbb{T}} \text {. }
$$

Proof. By Definition 30, $f \in L_{\Delta}^{1}\left([a, b]_{\mathbb{T}}, \mathbb{R}^{N}\right), f(t)=$ $\left(f^{1}(t), f^{2}(t), \ldots, f^{N}(t)\right)$ implies $f^{i} \in L_{\Delta}^{1}\left([a, b]_{\mathbb{T}}, \mathbb{R}\right)(i=$ $1,2, \ldots, N)$.

On one hand, If $f \equiv C=\left(C^{1}, C^{2}, \ldots, C^{N}\right) \Delta$-a.e. on $[a$, $b]_{\mathbb{T}}$, for any $h \in C_{a, b ; \mathrm{rd}}^{\alpha}\left([a, b]_{\mathbb{T}}, \mathbb{R}\right), h(t)=\left(h^{1}(t), h^{2}(t), \ldots\right.$, $\left.h^{N}(t)\right)$, Combining Theorem 35 with the definition of $C_{a, b ; \mathrm{rd}}^{\alpha}\left([a, b]_{\mathbb{T}}, \mathbb{R}\right)$, Definitions 30 and 32 , we have

$$
\begin{aligned}
\int_{a}^{b} & \left(f(t), T_{\alpha}(h)(t)\right) \Delta^{\alpha} t=\int_{a}^{b} \sum_{i=1}^{N} C^{i} T_{\alpha}\left(h^{i}\right)(t) \Delta^{\alpha} t \\
& =\sum_{i=1}^{N} \int_{a}^{b} C^{i} T_{\alpha}\left(h^{i}\right)(t) \Delta^{\alpha} t=\sum_{i=1}^{N} C^{i} \int_{a}^{b} T_{\alpha}(h)(t) \Delta^{\alpha} t \\
& =\sum_{i=1}^{N} C^{i}\left(h^{i}(b)-h^{i}(a)\right)=0 .
\end{aligned}
$$

On the other hand, $\forall h_{1} \in C_{a, b ; \mathrm{rd}}^{\alpha}\left([a, b]_{\mathbb{T}}, \mathbb{R}\right)$, let $h(t)=$ $\left(h_{1}(t), 0, \ldots, 0\right)$, then $h \in C_{a, b ; \mathrm{rd}}^{\alpha}\left([a, b]_{\mathbb{T}}, \mathbb{R}^{N}\right)$ and

$$
\int_{[a, b]_{\mathbb{T}}}\left(f(t), T_{\alpha}(h)(t)\right) \Delta^{\alpha} t=0 .
$$

That is

$$
\int_{[a, b]_{\mathbb{T}}} f^{1}(t) T_{\alpha}\left(h^{1}\right)(t) \Delta^{\alpha} t=0
$$

Because $C_{0, \mathrm{rd}}^{\alpha}\left([a, b]_{\mathbb{T}}, \mathbb{R}\right) \subset C_{a, b ; \mathrm{rd}}^{\alpha}\left([a, b]_{\mathbb{V}}, \mathbb{R}\right)$, by Theorem 29, there exists $C^{1} \in \mathbb{R}$ such that

$$
f^{1} \equiv C^{1} \quad \Delta \text {-a.e. on }[a, b]_{\mathbb{\pi}} \cdot
$$

Analogically, there exists $C^{i} \in \mathbb{R}(i=2,3, \ldots, N)$ such that

$$
f^{i} \equiv C^{i} \quad \Delta \text {-a.e. on }[a, b]_{\mathbb{T}} .
$$

Therefore,

$$
f \equiv\left(C^{1}, C^{2}, \ldots, C^{N}\right) \quad \Delta \text {-a.e. on }[a, b]_{\mathbb{T}} \cdot
$$

The proof is finished.

Now, we show the characterization of functions in $W_{\Delta ; a, b}^{\alpha, p}\left([a, b]_{\mathbb{T}}, \mathbb{R}^{N}\right)$ in terms of functions in $V_{\Delta ; a, b}^{\alpha, p}\left([a, b]_{\mathbb{T}}\right.$, $\left.\mathbb{R}^{N}\right)$.

Theorem 45. Assume that $u \in W_{\Delta ; a, b}^{\alpha, p}\left([a, b]_{\mathbb{T}}, \mathbb{R}^{N}\right)$ for some $p \in \mathbb{R}$ with $p \geq 1$, and that (63) holds for $g \in$ $L_{\alpha, \Delta}^{p}\left([a, b]_{\mathbb{T}}, \mathbb{R}^{N}\right)$. Then, there exists a unique function $x \in$ $V_{\Delta ; a, b}^{\alpha, p}\left([a, b]_{\mathbb{T}}, \mathbb{R}^{N}\right)$ such that

$$
\begin{aligned}
x & =\mathcal{u}, \\
T_{\alpha}(x) & =g \\
& \Delta \text {-a.e. on }[a, b]_{\mathbb{T}} .
\end{aligned}
$$

Proof. Let $\left\{e_{j}\right\}_{j=1}^{N}$ denote the canonical basis of $\mathbb{R}^{N}$. We can take $\phi \equiv e_{j}$ in (63), which guarantees

$$
\int_{[a, b]_{\mathbb{T}}}\left(g(t), e_{j}\right) \Delta^{\alpha} t=0 \quad(j=1,2, \ldots, N) .
$$

So, it is easy to get that

$$
\int_{[a, b]_{\mathbb{T}}} g(t) \Delta^{\alpha} t=0 .
$$

We define function $v:[a, b]_{\mathbb{T}} \rightarrow \mathbb{R}^{N}$ as

$$
v(t)=\int_{[a, b]_{\mathbb{T}}} g(s) \Delta^{\alpha} s .
$$

Equations (76) and (77) imply that $v \in V_{\Delta ; a, b}^{\alpha, p}\left([a, b]_{\mathbb{T}}, \mathbb{R}^{N}\right)$ and by Theorem 40 , we assert that

$$
\begin{aligned}
& \int_{[a, b]_{\mathbb{T}}}\left(v(t)-u(t), h^{\Delta}(t)\right) \Delta^{\alpha} t \\
& =-\int_{[a, b]_{\mathbb{T}}}\left(T_{\alpha}(v)(t)-g(t), h^{\sigma}(t)\right) \Delta^{\alpha} t
\end{aligned}
$$


for every $h \in C_{a, b ; r d}^{\alpha}\left([a, b]_{\mathbb{T}}, \mathbb{R}^{N}\right)$. It follows from Theorem 44 and (78) that there exists $C_{0} \in \mathbb{R}^{N}$ such that

$$
v-u \equiv C_{0}, \quad \text { on }[a, b]_{\mathbb{T}} .
$$

As a result of the fundamental theorem of conformable fractional calculus we can choose the function $x:[a, b]_{\mathbb{T}} \rightarrow$ $\mathbb{R}^{N}$ defined as

$$
x(t)=v(t)-C_{0}, \quad t \in[a, b]_{\mathbb{T}} .
$$

Then, the function $x$ is the unique function in $V_{\Delta ; a, b}^{\alpha, p}\left([a, b]_{\mathbb{T}}, \mathbb{R}^{N}\right)$ for which (74) is valid. The proof is complete.

If we identify $u \in W_{\Delta ; a, b}^{\alpha, p}\left([a, b]_{\mathbb{T}}, \mathbb{R}^{N}\right)$ with its absolutely continuous representative $x \in V_{\Delta ; a, b}^{\alpha, p}\left([a, b]_{\mathbb{T}}, \mathbb{R}^{N}\right)$ for which (74) holds, then the set $W_{\Delta ; a, b}^{\alpha, p}\left([a, b]_{\mathbb{T}}, \mathbb{R}^{N}\right)$ can be endowed with the structure of Banach space.

Theorem 46. Suppose $p \in \mathbb{R}$ and $p \geq 1$. Then the set $W_{\Delta ; a, b}^{\alpha, p}\left([a, b]_{\mathbb{T}}, \mathbb{R}^{N}\right)$ is a Banach space together with the norm defined as

$$
\begin{aligned}
& \|u\|_{W_{\Delta ; a, b}^{\alpha, p}} \\
& =\left(\int_{[a, b]_{\mathbb{T}}}\left|u^{\sigma}(t)\right|^{p} \Delta^{\alpha} t+\int_{[a, b]_{\mathbb{T}}}\left|T_{\alpha}(u)(t)\right|^{p} \Delta^{\alpha} t\right)^{1 / p}, \\
& \forall u \in W_{\Delta ; a, b}^{\alpha, p}\left([a, b]_{\mathbb{T}}, \mathbb{R}^{N}\right) .
\end{aligned}
$$

Moreover, the set $H_{\Delta ; a, b}^{\alpha}=W_{\Delta ; a, b}^{\alpha, 2}\left([a, b]_{\mathbb{T}}, \mathbb{R}^{N}\right)$ is a Hilbert space together with the inner product

$$
\begin{aligned}
\langle u, v\rangle_{H_{\Delta ; a, b}^{\alpha}}=\int_{[a, b]_{\top}}\left(u^{\sigma}(t), v^{\sigma}(t)\right) \Delta^{\alpha} t & \\
& +\int_{[a, b]_{\top}}\left(T_{\alpha}(u)(t), T_{\alpha}(v)(t)\right) \Delta^{\alpha} t, \\
& \forall u, v \in H_{\Delta ; a, b}^{1} .
\end{aligned}
$$

Proof. Let $\left\{u_{n}\right\}_{n \in \mathbb{N}}$ be a Cauchy sequence in $W_{\Delta ; a, b}^{\alpha, p}\left([a, b]_{\mathbb{T}}\right.$, $\left.\mathbb{R}^{N}\right)$. According to the definition of $W_{\Delta ; a, b}^{\alpha, p}\left([a, b]_{\mathbb{T}}, \mathbb{R}^{N}\right)$, we have $\left\{u_{n}\right\}_{n \in \mathbb{N}} \subset L_{\alpha, \Delta}^{p}\left([a, b]_{\mathbb{T}}, \mathbb{R}^{N}\right)$ and there exist $g_{n}$ : $[a, b]^{\kappa} \rightarrow \mathbb{R}^{N}$ such that $\left\{g_{n}\right\}_{n \in \mathbb{N}} \subset L_{\alpha, \Delta}^{p}\left([a, b]_{\mathbb{T}}, \mathbb{R}^{N}\right)$ and

$$
\begin{aligned}
& \int_{[a, b]_{\mathbb{T}}}\left(u_{n}(t), T_{\alpha}(\phi)(t)\right) \Delta^{\alpha} t \\
& =-\int_{[a, b]_{\mathbb{T}}}\left(g_{n}(t), \phi^{\sigma}(t)\right) \Delta^{\alpha} t, \\
& \forall \phi \in C_{a, b ; \mathrm{rd}}^{\alpha}\left([a, b]_{\mathbb{T}}, \mathbb{R}^{N}\right) .
\end{aligned}
$$

Therefore, by Theorem 45, there exists $\left\{x_{n}\right\}_{n \in \mathbb{N}} \quad \mathrm{C}$ $V_{\Delta ; a, b}^{\alpha, p}\left([a, b]_{\mathbb{T}}, \mathbb{R}^{N}\right)$ such that

$$
\begin{aligned}
x_{n} & =u_{n}, \\
T_{\alpha}\left(x_{n}\right) & =g_{n}
\end{aligned}
$$

$\Delta$-a.e. on $[a, b]_{\mathbb{T}}$.

Equations (83) and (84) imply that

$$
\begin{aligned}
& \int_{[a, b]_{\mathbb{T}}}\left(x_{n}(t), T_{\alpha}(\phi)(t)\right) \Delta^{\alpha} t \\
& =-\int_{[a, b]_{\mathbb{T}}}\left(T_{\alpha}\left(x_{n}\right)(t), \phi^{\sigma}(t)\right) \Delta^{\alpha} t
\end{aligned}
$$

for all $\phi \in C_{a, b ; \mathrm{rd}}^{\alpha}\left([a, b]_{\mathbb{T}}, \mathbb{R}^{N}\right)$. On account of $\left\{u_{n}\right\}_{n \in \mathbb{N}}$ is a Cauchy sequence in $W_{\Delta ; a, b}^{\alpha, p}\left([a, b]_{\mathbb{T}}, \mathbb{R}^{N}\right)$, by $(81)$, we can get

$$
\begin{aligned}
& \int_{[a, b]_{\pi}}\left|u_{n}^{\sigma}(t)-u_{m}^{\sigma}(t)\right|^{p} \Delta^{\alpha} t \longrightarrow 0 \\
&(m, n \longrightarrow \infty) \\
& \int_{[a, b]_{\pi}}\left|T_{\alpha}\left(u_{n}\right)(t)-T_{\alpha}\left(u_{m}\right)(t)\right|^{p} \Delta^{\alpha} t \longrightarrow 0 \\
&(m, n \longrightarrow \infty) .
\end{aligned}
$$

It follows from Theorems 33 and 35 and (86) and (87) that

$$
\begin{aligned}
& \int_{[a, b]_{\top}}\left|u_{n}(t)-u_{m}(t)\right|^{p} \Delta^{\alpha} t=\int_{[a, b]_{\top}} \mid\left(u_{n}^{\sigma}(t)-u_{m}^{\sigma}(t)\right) \\
& -\left.\mu(t) t^{\alpha-1}\left(T_{\alpha}(u)_{n}(t)-T_{\alpha}(u)_{m}(t)\right)\right|^{p} \Delta^{\alpha} t \\
& \leq 2^{p-1} \int_{[a, b]_{\top}}\left|u_{n}^{\sigma}(t)-u_{m}^{\sigma}(t)\right|^{p} \Delta^{\alpha} t \\
& +2^{p-1} a^{p(\alpha-1)}(\sigma(b)-a)^{p} \int_{[a, b]_{\top}} \mid T_{\alpha}(u)_{n}(t) \\
& -\left.T_{\alpha}(u)_{m}(t)\right|^{p} \Delta^{\alpha} t \longrightarrow 0 \quad(m, n \longrightarrow \infty) .
\end{aligned}
$$

For another, from Theorem 41, (87), and (88), there exist $u, g \in L_{\alpha, \Delta}^{p}\left([a, b]_{\mathbb{J}}, \mathbb{R}^{N}\right)$ such that

$$
\begin{aligned}
\left\|u_{n}-u\right\|_{L_{\alpha, \Delta}^{p}} \longrightarrow 0 & (n \longrightarrow \infty), \\
\left\|T_{\alpha}(u)_{n}-g\right\|_{L_{\alpha, \Delta}^{p}} \longrightarrow 0 & (n \longrightarrow \infty) .
\end{aligned}
$$


Combining (85) with (89), one has

$$
\begin{aligned}
& \int_{[a, b]_{\mathbb{T}}}\left(u(t), T_{\alpha}(\phi)(t)\right) \Delta^{\alpha} t \\
& =-\int_{[a, b]_{\mathbb{T}}}\left(g(t), \phi^{\sigma}(t)\right) \Delta^{\alpha} t
\end{aligned}
$$

for all $\phi \in C_{a, b ; \text { rd }}^{\alpha}\left([a, b]_{\mathbb{T}}, \mathbb{R}^{N}\right)$. By (90), we claim that $u \in$ $W_{\Delta ; a, b}^{\alpha, p}\left([a, b]_{\mathbb{T}}, \mathbb{R}^{N}\right)$. Furthermore, it follows from Theorems 33 and 35 and (89) that

$$
\begin{aligned}
& \int_{[a, b]_{\mathbb{T}}}\left|u_{n}^{\sigma}(t)-u^{\sigma}(t)\right|^{p} \Delta^{\alpha} t=\int_{[a, b]_{\mathbb{T}}} \mid\left(u_{n}(t)-u(t)\right) \\
& +\left.\mu(t) t^{\alpha-1}\left(T_{\alpha}\left(u_{n}\right)(t)-T_{\alpha}(u)(t)\right)\right|^{p} \Delta^{\alpha} t \\
& \quad=\int_{[a, b]_{\mathbb{T}}} \mid\left(u_{n}(t)-u(t)\right) \\
& +\left.\mu(t) t^{\alpha-1}\left(T_{\alpha}\left(u_{n}\right)(t)-g(t)\right)\right|^{p} \Delta^{\alpha} t \\
& \quad \leq 2^{p-1} \int_{[a, b]_{\mathbb{T}}}\left|u_{n}(t)-u(t)\right|^{p} \Delta^{\alpha} t \\
& +2^{p-1} a^{p(\alpha-1)}(\sigma(b)-a)^{p} \int_{[a, b]_{\mathbb{T}}} \mid T_{\alpha}(u)_{n}(t) \\
& -\left.g(t)\right|^{2} \Delta^{\alpha} t \longrightarrow 0 \quad(n \longrightarrow \infty) .
\end{aligned}
$$

Hence, by Remark 43, (89), (91), and Theorem 45, there exists $x \in V_{\Delta ; a, b}^{\alpha, p}\left([a, b]_{\mathbb{T}}, \mathbb{R}^{N}\right) \subset W_{\Delta ; a, b}^{\alpha, p}\left([a, b]_{\mathbb{T}}, \mathbb{R}^{N}\right)$ such that

$$
\left\|u_{n}-x\right\|_{W_{\Delta ; a, b}^{\alpha, p}} \longrightarrow 0 \quad(n \longrightarrow \infty)
$$

So, $W_{\Delta ; a, b}^{\alpha, p}\left([a, b]_{\mathbb{T}}, \mathbb{R}^{N}\right)$ is a Banach space.

Clearly, the set $H_{\Delta ; a, b}^{\alpha}$ is a Hilbert space together with the inner product

$$
\begin{array}{r}
\langle u, v\rangle_{H_{\Delta ; a, b}^{\alpha}}^{\alpha}=\int_{[a, b]_{\mathbb{T}}}\left(u^{\sigma}(t), v^{\sigma}(t)\right) \Delta^{\alpha} t \\
+\int_{[a, b]_{\mathbb{T}}}\left(T_{\alpha}(u)(t), T_{\alpha}(v)(t)\right) \Delta^{\alpha} t \\
\forall u, v \in H_{\Delta ; a, b}^{\alpha} .
\end{array}
$$

The proof is finished.

Next, we will show some important properties of the Banach space $W_{\Delta ; a, b}^{\alpha, p}\left([a, b]_{\mathbb{T}}, \mathbb{R}^{N}\right)$.

Theorem 47. The space $W_{\Delta ; a, b}^{\alpha, p}\left([a, b]_{\mathbb{T}}, \mathbb{R}^{N}\right)$ is a reflexive and uniformly convex Banach space when $p \in(1,+\infty)$.
Proof. By Clarkson inequality, we have

$$
\begin{aligned}
& \left\|\frac{1}{2}\left(u^{\sigma}+v^{\sigma}\right)\right\|_{L_{\alpha, \Delta}^{p}}^{p}+\left\|\frac{1}{2}\left(u^{\sigma}-v^{\sigma}\right)\right\|_{L_{\alpha, \Delta}^{p}}^{p} \\
& \quad \leq \frac{1}{2}\left\|u^{\sigma}\right\|_{L_{\alpha, \Delta}^{p}}^{p}+\frac{1}{2}\left\|v^{\sigma}\right\|_{L_{\alpha, \Delta}^{p}}^{p}, \quad p \in[2,+\infty), \\
& \left\|\frac{1}{2}\left(T_{\alpha}(u)+T_{\alpha}(v)\right)\right\|_{L_{\alpha, \Delta}^{p}}^{p}+\left\|\frac{1}{2}\left(T_{\alpha}(u)-T_{\alpha}(v)\right)\right\|_{L_{\alpha, \Delta}^{p}}^{p} \\
& \quad \leq \frac{1}{2}\left\|T_{\alpha}(u)\right\|_{L_{\alpha, \Delta}^{p}}^{p}+\frac{1}{2}\left\|T_{\alpha}(v)\right\|_{L_{\alpha, \Delta}^{p}}^{p}, p \in[2,+\infty), \\
& \left\|\frac{1}{2}\left(u^{\sigma}+v^{\sigma}\right)\right\|_{L_{\alpha, \Delta}^{p}}^{q}+\left\|\frac{1}{2}\left(u^{\sigma}-v^{\sigma}\right)\right\|_{L_{\alpha, \Delta}^{p}}^{q}, p \in(1,2], \\
& \quad \leq\left(\frac{1}{2}\left\|u^{\sigma}\right\|_{L_{\alpha, \Delta}^{p}}^{p}+\frac{1}{2}\left\|v^{\sigma}\right\|_{L_{\alpha, \Delta}}^{p}\right)^{q-1}, p \in(1,2], \\
& \quad \leq\left(\frac{1}{2}\left\|T_{\alpha}(u)\right\|_{L_{\alpha, \Delta}}^{p}+\frac{1}{2}\left\|T_{\alpha}(v)\right\|_{L_{\alpha, \Delta}}^{p}\right)^{q-1}, \\
& \qquad\left(T_{\alpha}(u)+T_{\alpha}(v)\right)\left\|_{L_{\alpha, \Delta}^{p}}^{q}+\right\| \frac{1}{2}\left(T_{\alpha}(u)-T_{\alpha}(v)\right) \|_{L_{\alpha, \Delta}^{p}}^{q}
\end{aligned}
$$

where $1 / p+1 / q=1$. Thus, $\forall \epsilon>0$, for any $u, v \epsilon$ $W_{\Delta ; a, b}^{\alpha, p}\left([a, b]_{\mathbb{T}}, \mathbb{R}^{N}\right),\|u\|_{W_{\Delta ; a, b}^{\alpha, p}}=\|v\|_{W_{\Delta ; a, b}^{\alpha, p}}=1$ and $\|u-v\|_{W_{\Delta ; a, b}^{\alpha, p}} \geq$ $\epsilon$, if $2 \leq p<+\infty$, by (94) and (95), one has

$$
\begin{aligned}
\left\|\frac{1}{2}(u+v)\right\|_{W_{\Delta ; a, b}^{1, p}=}^{p}= & \frac{1}{2}\left(u^{\sigma}+v^{\sigma}\right) \|_{L_{\alpha, \Delta}^{p}}^{p} \\
& +\left\|\frac{1}{2}\left(T_{\alpha}(u)+T_{\alpha}(v)\right)\right\|_{L_{\alpha, \Delta}^{p}}^{p} \\
\leq & \frac{1}{2}\left\|u^{\sigma}\right\|_{L_{\alpha, \Delta}^{p}}^{p}+\frac{1}{2}\left\|v^{\sigma}\right\|_{L_{\alpha, \Delta}^{p}}^{p} \\
& +\frac{1}{2}\left\|T_{\alpha}(u)\right\|_{L_{\alpha, \Delta}^{p}}^{p}+\frac{1}{2}\left\|T_{\alpha}(v)\right\|_{L_{\alpha, \Delta}^{p}}^{p} \\
& -\left\|\frac{1}{2}\left(u^{\sigma}-v^{\sigma}\right)\right\|_{L_{\alpha, \Delta}^{p}}^{p} \\
& -\left\|\frac{1}{2}\left(T_{\alpha}(u)-T_{\alpha}(v)\right)\right\|_{L_{\alpha, \Delta}^{p}}^{p} \\
= & \frac{1}{2}\|u\|_{W_{\Delta ; a, b}^{1, p}}^{p}+\frac{1}{2}\|v\|_{W_{\Delta ; a, b}^{1, p}}^{p} \\
& -\left\|\frac{1}{2}(u-v)\right\|_{W_{\Delta ; a, b}^{1, p}}^{p} \leq 1-\left(\frac{\epsilon}{2}\right)^{p} ;
\end{aligned}
$$


12

Advances in Mathematical Physics

if $p \in(1,2]$, by (96) and (97), we have

$$
\begin{aligned}
\| & \frac{1}{2}(u+v) \|_{W_{\Delta ; a, b}^{1, p}}^{q}=\left(\left\|\frac{1}{2}(u+v)\right\|_{W_{\Delta ; a, b}^{1, p}}^{p}\right)^{q / p} \\
& =\left(\left\|\frac{1}{2}(u+v)\right\|_{W_{\Delta ; a, b}^{1, p}}^{p}\right)^{q-1}=\left(\left\|\frac{1}{2}\left(u^{\sigma}+v^{\sigma}\right)\right\|_{L_{\alpha, \Delta}^{p}}^{p}\right. \\
& \left.+\left\|\frac{1}{2}\left(T_{\alpha}(u)+T_{\alpha}(v)\right)\right\|_{L_{\alpha, \Delta}^{p}}^{p}\right)^{q-1} \\
& =\left\|\frac{1}{2}\left(u^{\sigma}+v^{\sigma}\right)\right\|_{L_{\alpha, \Delta}^{p}}^{q}+\left\|\frac{1}{2}\left(T_{\alpha}(u)+T_{\alpha}(v)\right)\right\|_{L_{\alpha, \Delta}^{p}}^{q} \\
& \leq\left(\frac{1}{2}\left\|u^{\sigma}\right\|_{L_{\alpha, \Delta}^{p}}^{p}+\frac{1}{2}\left\|v^{\sigma}\right\|_{L_{\alpha, \Delta}^{p}}^{p}\right)^{q-1}+\left(\frac{1}{2}\left\|T_{\alpha}(u)\right\|_{L_{\alpha, \Delta}^{p}}^{p}\right. \\
& \left.+\frac{1}{2}\left\|T_{\alpha}(v)\right\|_{L_{\alpha, \Delta}^{p}}^{p}\right)^{q-1}-\left\|\frac{1}{2}\left(u^{\sigma}-v^{\sigma}\right)\right\|_{L_{\alpha, \Delta}^{p}}^{q} \\
& -\left\|\frac{1}{2}\left(T_{\alpha}(u)-T_{\alpha}(v)\right)\right\|_{L_{\alpha, \Delta}^{p}}^{q} \leq 2-\left(\frac{\epsilon}{2}\right)^{q} .
\end{aligned}
$$

Combining (98) with (99), $W_{\Delta ; a, b}^{\alpha, p}\left([a, b]_{\mathbb{T}}, \mathbb{R}^{N}\right)$ is a uniformly convex Banach space. By functional analysis, we know that a uniformly convex Banach space is reflexive. The proof is complete.

Theorem 48. There exists $K=K(p)>0$ such that the inequality

$$
\|u\|_{\infty} \leq K\|u\|_{W_{\Delta ; a, b}^{\alpha, p}}
$$

holds for all $u \in W_{\Delta ; a, b}^{\alpha, p}\left([a, b]_{\mathbb{T}}, \mathbb{R}^{N}\right)$, where $\|u\|_{\infty}=$ $\max _{t \in[0, T]_{\mathbb{T}}}|u(t)|$.

Moreover, if $\int_{[a, b]_{\pi}} u(t) \Delta^{\alpha} t=0$, then

$$
\|u\|_{\infty} \leq K\left\|T_{\alpha}(u)\right\|_{L_{\alpha, \Delta}^{p}} .
$$

Proof. Going to the components of $u$, we can suppose that $N=1$. If $u \in W_{\Delta ; a, b}^{\alpha, p}\left([a, b]_{\mathbb{T}}, \mathbb{R}^{N}\right)$, from Theorem $45, U(t)=$ $\int_{[a, t]_{\mathbb{T}}} u(s) \Delta^{\alpha} s$ is absolutely continuous on $[a, b]_{\mathbb{T}}$. On the basis of Theorem 15 , there exists $\zeta \in[a, b]_{\mathbb{I}}$ such that

$$
\begin{aligned}
u(\zeta) & \leq \frac{U(b)-U(a)}{b-a} \max \left\{b^{1-\alpha}, 1\right\} \\
& =\frac{\max \left\{b^{1-\alpha}, 1\right\}}{b-a} \int_{[a, b]_{\pi}} u(s) \Delta^{\alpha} s .
\end{aligned}
$$

Hence, for $t \in[a, b]_{\mathbb{T}}$, using Theorems 15 and 26 and (102), we obtain

$$
\begin{aligned}
& |u(t)|=\left|u(\zeta)+\int_{[\zeta, t)_{\mathbb{T}}} T_{\alpha}(u)(s) \Delta^{\alpha} s\right| \leq|u(\zeta)| \\
& \quad+\int_{[a, b]_{\mathbb{T}}}\left|T_{\alpha}(u)(s)\right| \Delta^{\alpha} s \\
& \quad \leq \frac{\max \left\{b^{1-\alpha}, 1\right\}}{b-a}\left|\int_{[a, b]_{\mathbb{T}}} u(s) \Delta^{\alpha} s\right| \\
& \quad+\left[\max \left\{a^{\alpha-1}, 1\right\}(b-a)\right]^{1 / q} \\
& \quad \cdot\left(\int_{[a, b]_{\mathbb{T}}}\left|T_{\alpha}(u)(s)\right|^{p} \Delta^{\alpha} s\right)^{1 / p},
\end{aligned}
$$

where $1 / p+1 / q=1$. If $\int_{[a, b]_{\top}} u(t) \Delta^{\alpha} t=0$, by (103), (101) holds. In the general case, for $t \in[a, b]_{\mathbb{T}}$, from Theorem 33 and Hölder's inequality, we have

$$
\begin{aligned}
& |u(t)| \leq \frac{\max \left\{b^{1-\alpha}, 1\right\}}{b-a}\left|\int_{[a, b]_{\pi}} u(s) \Delta^{\alpha} s\right| \\
& +\left[\max \left\{a^{\alpha-1}, 1\right\}(b-a)\right]^{1 / q} \\
& \cdot\left(\int_{[a, b]_{\mathbb{T}}}\left|T_{\alpha}(u)(s)\right|^{p} \Delta^{\alpha} s\right)^{1 / p} \leq \frac{\max \left\{b^{1-\alpha}, 1\right\}}{b-a} \\
& \cdot \int_{[a, b]_{\mathbb{T}}}|u(s)| \Delta^{\alpha} s+\left[\max \left\{a^{\alpha-1}, 1\right\}(b-a)\right]^{1 / q} \\
& \cdot\left(\int_{[a, b]_{\mathbb{T}}}\left|T_{\alpha}(u)(s)\right|^{p} \Delta^{\alpha} s\right)^{1 / p}=\frac{\max \left\{b^{1-\alpha}, 1\right\}}{b-a} \\
& \cdot \int_{[a, b]_{\mathbb{T}}}\left|u^{\sigma}(s)-\mu(s) s^{\alpha-1} T_{\alpha}(u)(s)\right| \Delta^{\alpha} s \\
& +\left[\max \left\{a^{\alpha-1}, 1\right\}(b-a)\right]^{1 / q} \\
& \cdot\left(\int_{[a, b]_{\mathbb{T}}}\left|T_{\alpha}(u)(s)\right|^{p} \Delta^{\alpha} s\right)^{1 / p} \leq \frac{\max \left\{b^{1-\alpha}, 1\right\}}{b-a} \\
& \cdot \int_{[a, b]_{\mathbb{T}}}\left|u^{\sigma}(s)\right| \Delta^{\alpha} s+a^{\alpha-1}(\sigma(b)-a) \\
& \frac{\max \left\{b^{1-\alpha}, 1\right\}}{b-a} \int_{[a, b]_{\mathbb{T}}}\left|T_{\alpha}(u)(s)\right| \Delta^{\alpha} s \\
& +\left[\max \left\{a^{\alpha-1}, 1\right\}(b-a)\right]^{1 / q} \\
& \cdot\left(\int_{[a, b]_{\mathbb{T}}}\left|T_{\alpha}(u)(s)\right|^{p} \Delta^{\alpha} s\right)^{1 / p}
\end{aligned}
$$




$$
\begin{aligned}
& \leq \frac{\max \left\{b^{1-\alpha}, 1\right\}}{b-a}\left[\max \left\{a^{\alpha-1}, 1\right\}(b-a)\right]^{1 / q} \\
& \cdot\left(\int_{[a, b]_{\top}}\left|u^{\sigma}(s)\right|^{p} \Delta^{\alpha} s\right)^{1 / p}+a^{\alpha-1}(\sigma(b)-a) \\
& \cdot \frac{\max \left\{b^{1-\alpha}, 1\right\}}{b-a}\left[\max \left\{a^{\alpha-1}, 1\right\}(b-a)\right]^{1 / q} \\
& \cdot\left(\int_{[a, b]_{\top}}\left|T_{\alpha}(u)(s)\right|^{p} \Delta^{\alpha} s\right)^{1 / p} \\
& +\left[\max \left\{a^{\alpha-1}, 1\right\}(b-a)\right]^{1 / q} \\
& \cdot\left(\int_{[a, b]_{\top}}\left|T_{\alpha}(u)(s)\right|^{p} \Delta^{\alpha} s\right)^{1 / p} \leq K\|u\|_{W_{\Delta ; a, b}^{\alpha, p},}
\end{aligned}
$$

where $K=\left(\max \left\{b^{1-\alpha}, 1\right\} /(b-a)+a^{\alpha-1}(\sigma(b)-\right.$ $\left.a)\left(\max \left\{b^{1-\alpha}, 1\right\} /(b-a)\right)+1\right)\left[\max \left\{a^{\alpha-1}, 1\right\}(b-a)\right]^{1 / q}$. By (104), (100) holds. The proof is complete.

Remark 49. It follows from Theorem 48 that $W_{\Delta ; a, b}^{\alpha, p}\left([a, b]_{\mathbb{T}}, \mathbb{R}^{N}\right) \quad$ is continuously immersed into $C\left([a, b]_{\mathbb{T}}, \mathbb{R}^{N}\right)$ with the norm $\|\cdot\|_{\infty}$.

Theorem 50. If the sequence $\left\{u_{k}\right\}_{k \in \mathbb{N}} \subset W_{\Delta ; a, b}^{\alpha, p}\left([a, b]_{\mathbb{T}}, \mathbb{R}^{N}\right)$ converges weakly to $u$ in $W_{\Delta ; a, b}^{\alpha, p}\left([a, b]_{\mathbb{T}}, \mathbb{R}^{N}\right)$, then $\left\{u_{k}\right\}_{k \in \mathbb{N}}$ converges strongly in $C\left([a, b]_{\mathbb{T}}, \mathbb{R}^{N}\right)$ to $u$.

Proof. Owing to $u_{k} \rightarrow u$ in $W_{\Delta ; a, b}^{\alpha, p}\left([a, b]_{\mathbb{T}}, \mathbb{R}^{N}\right),\left\{u_{k}\right\}_{k \in \mathbb{N}}$ is bounded in $W_{\Delta ; a, b}^{\alpha, p}\left([a, b]_{\mathbb{T}}, \mathbb{R}^{N}\right)$ and, hence, in $C\left([a, b]_{\mathbb{T}}, \mathbb{R}^{N}\right)$. It follows from Remark 49 that $u_{k} \rightarrow u$ in $C\left([a, b]_{\mathbb{T}}, \mathbb{R}^{N}\right)$. For $t_{1}, t_{2} \in[a, b]_{\mathbb{T}}, t_{1} \leq t_{2}$, there exists $C_{1}>0$ such that

$$
\begin{aligned}
& \left|u_{k}\left(t_{2}\right)-u_{k}\left(t_{1}\right)\right| \leq \int_{\left[t_{1}, t_{2}\right]_{\top}}\left|T_{\alpha}\left(u_{k}\right)(s)\right| \Delta^{\alpha} s \\
& \leq\left[\max \left\{t_{1}^{\alpha-1}, 1\right\}\left(t_{2}-t_{1}\right)\right]^{1 / q} \\
& \cdot\left(\int_{\left[t_{1}, t_{2}\right]_{\top}}\left|T_{\alpha}\left(u_{k}\right)(s)\right|^{p} \Delta^{\alpha} s\right)^{1 / p} \\
& \leq\left(t_{2}-t_{1}\right)^{1 / q} \max \left\{t_{1}^{\alpha-1}, 1\right\}^{1 / q}\left\|u_{k}\right\|_{W_{\Delta ; a, b}^{\alpha, p}} \\
& \leq C_{1}\left(t_{2}-t_{1}\right)^{1 / q} .
\end{aligned}
$$

In view of this, the sequence $\left\{u_{k}\right\}_{k \in \mathbb{N}}$ is equicontinuous. According to Ascoli-Arzela theorem, $\left\{u_{k}\right\}_{k \in \mathbb{N}}$ is relatively in $C\left([a, b]_{\mathbb{T}}, \mathbb{R}^{N}\right)$. By the uniqueness of the weak limit in $C\left([a, b]_{\mathbb{V}}, \mathbb{R}^{N}\right)$, every uniformly convergent subsequence of $\left\{u_{k}\right\}_{k \in \mathbb{N}}$ converges to $u$. Consequently, $\left\{u_{k}\right\}_{k \in \mathbb{N}}$ converges strongly in $C\left([a, b]_{\mathbb{T}}, \mathbb{R}^{N}\right)$ to $u$. The proof is complete.

Remark 51. It follows from Theorem 50 that $W_{\Delta ; a, b}^{\alpha, p}([a$, $\left.b]_{\mathbb{V}}, \mathbb{R}^{N}\right)$ is compact immersed into $C\left([a, b]_{\mathbb{T}}, \mathbb{R}^{N}\right)$ with the norm $\|\cdot\|_{\infty}$.
Theorem 52. Let $L:[a, b]_{\mathbb{T}} \times \mathbb{R}^{N} \times \mathbb{R}^{N} \rightarrow \mathbb{R},(t, x, y) \rightarrow$ $L(t, x, y)$ be $\Delta$-measurable in $t$ for each $(x, y) \in \mathbb{R}^{N} \times$ $\mathbb{R}^{N}$ and continuously conformal fractional differentiable of order $\alpha$ in $(x, y)$ for $\Delta$-almost every $t \in[0, T]_{\mathbb{T}}$. If there exist $\widehat{a} \in C\left(\mathbb{R}^{+}, \mathbb{R}^{+}\right), \widehat{b} \in L_{\alpha, \Delta}^{1}\left([a, b]_{\mathbb{T}}, \mathbb{R}^{+}\right)$and $\hat{c} \in$ $L_{\alpha, \Delta}^{q}\left([a, b]_{\mathbb{T}}, \mathbb{R}^{+}\right)(1<q<+\infty)$ such that for $\Delta$-almost $t \in$ $[a, b]_{\mathbb{T}}$ and every $(x, y) \in \mathbb{R}^{N} \times \mathbb{R}^{N}$, one has

$$
\begin{gathered}
|L(t, x, y)| \leq \widehat{a}(|x|)\left(\widehat{b}(t)+|y|^{p}\right), \\
\left|L_{x}(t, x, y)\right| \leq \widehat{a}(|x|)\left(\widehat{b}(t)+|y|^{p}\right), \\
\left|L_{y}(t, x, y)\right| \leq \widehat{a}(|x|)\left(\widehat{c}(t)+|y|^{p-1}\right),
\end{gathered}
$$

where $1 / p+1 / q=1$, then the functional $\Phi$ : $W_{\Delta ; a, b}^{\alpha, p}\left([a, b]_{\mathbb{T}}, \mathbb{R}^{N}\right) \rightarrow \mathbb{R}$ defined by

$$
\Phi(u)=\int_{[a, b]_{\top}} L\left(\sigma(t), u^{\sigma}(t), T_{\alpha}(u)(t)\right) \Delta^{\alpha} t
$$

is continuously differentiable on $W_{\Delta ; a, b}^{\alpha, p}\left([a, b]_{\mathbb{T}}, \mathbb{R}^{N}\right)$ and

$$
\begin{aligned}
& \left\langle\Phi^{\prime}(u), v\right\rangle=\int_{[a, b]_{\top}}\left(L_{x}\left(\sigma(t), u^{\sigma}(t), T_{\alpha}(u)(t)\right),\right. \\
& \left.v^{\sigma}(t)\right) \Delta^{\alpha} t \\
& +\int_{[a, b]_{\top}}\left(L_{y}\left(\sigma(t), u^{\sigma}(t), T_{\alpha}(u)(t)\right), T_{\alpha}(v)(t)\right) \\
& \cdot \Delta^{\alpha} t .
\end{aligned}
$$

Proof. It suffices to prove that $\Phi$ has at every point $u$ a directional derivative $\Phi^{\prime}(u) \in\left(W_{\Delta ; a, b}^{\alpha, p}\left([a, b]_{\mathbb{T}}, \mathbb{R}^{N}\right)\right)^{*}$ given by (108) and that the mapping

$$
\begin{gathered}
\Phi^{\prime}: W_{\Delta ; a, b}^{\alpha, p}\left([a, b]_{\mathbb{T}}, \mathbb{R}^{N}\right) \longrightarrow \\
\left(W_{\Delta ; a, b}^{\alpha, p}\left([a, b]_{\mathbb{T}}, \mathbb{R}^{N}\right)\right)^{*}
\end{gathered}
$$

is continuous.

As a matter of fact, on one hand, it follows from (106) that $\Phi$ is everywhere finite on $W_{\Delta ; a, b}^{\alpha, p}\left([a, b]_{\mathbb{T}}, \mathbb{R}^{N}\right)$. We denote, for $u$ and $v$ fixed in $W_{\Delta ; a, b}^{\alpha, p}\left([a, b]_{\mathbb{T}}, \mathbb{R}^{N}\right), t \in[a, b]_{\mathbb{J}}, \lambda \in[-1,1]$,

$$
\begin{aligned}
& G(\lambda, t) \\
& =L\left(\sigma(t), u^{\sigma}(t)+\lambda v^{\sigma}(t), T_{\alpha}(u)(t)+\lambda T_{\alpha}(v)(t)\right), \\
& \Psi(\lambda)=\int_{[a, b]_{\top}} G(\lambda, t) \Delta^{\alpha} t=\Phi(u+\lambda v) .
\end{aligned}
$$


In the light of (106), we obtain

$$
\begin{aligned}
& \left|D_{\lambda} G(\lambda, t)\right| \leq \mid\left(D _ { x } L \left(\sigma(t), u^{\sigma}(t)\right.\right. \\
& \left.\left.+\lambda v^{\sigma}(t), T_{\alpha}(u)(t)+\lambda T_{\alpha}(v)(t)\right), v^{\sigma}(t)\right) \mid \\
& +\mid\left(D _ { y } L \left(\sigma(t), u^{\sigma}(t)+\lambda v^{\sigma}(t), T_{\alpha}(u)(t)\right.\right. \\
& \left.\left.+\lambda T_{\alpha}(v)(t)\right), T_{\alpha}(v)(t)\right) \mid \leq \widehat{a}\left(\left|u^{\sigma}(t)+\lambda v^{\sigma}(t)\right|\right) \\
& +\left(\widehat{b}^{\sigma}(t)+\left|T_{\alpha}(u)(t)+\lambda T_{\alpha}(v)(t)\right|^{p}\right)\left|v^{\sigma}(t)\right| \\
& +\widehat{a}\left(\left|u^{\sigma}(t)+\lambda v^{\sigma}(t)\right|\right)\left(\widehat{c}^{\sigma}(t)+\mid T_{\alpha}(u)(t)\right. \\
& \left.+\left.\lambda T_{\alpha}(v)(t)\right|^{p-1}\right)\left|T_{\alpha}(v)(t)\right| \leq \bar{a}\left(\widehat{b}^{\sigma}(t)\right. \\
& \left.+\left(\left|T_{\alpha}(u)(t)\right|+\left|T_{\alpha}(v)(t)\right|\right)^{p}\right)\left|v^{\sigma}(t)\right|+\bar{a}\left(\widehat{c}^{\sigma}(t)\right. \\
& \left.+\left(\left|T_{\alpha}(u)(t)\right|+\left|T_{\alpha}(v)(t)\right|\right)^{p-1}\right)\left|T_{\alpha}(v)(t)\right| \triangleq d(t),
\end{aligned}
$$

where

$$
\bar{a}=\max _{(\lambda, t) \in[-1,1] \times[a, b]_{\mathbb{T}}} \widehat{a}(|u(t)+\lambda v(t)|) .
$$

By the definition of function $d, d \in L_{\alpha, \Delta}^{1}\left([a, b]_{\mathbb{V}}, \mathbb{R}^{+}\right)$. Since $\widehat{b} \in L_{\alpha, \Delta}^{1}\left([a, b]_{\mathbb{T}}, \mathbb{R}^{+}\right),\left(\left|T_{\alpha}(u)\right|+\left|T_{\alpha}(v)\right|\right)^{p} \in L_{\alpha, \Delta}^{1}([a$, $\left.b]_{\mathbb{T}}, \mathbb{R}\right), \widehat{c} \in L_{\alpha, \Delta}^{q}\left([a, b]_{\mathbb{T}}, \mathbb{R}^{+}\right)$, one has

$$
\begin{aligned}
& \left|D_{\lambda} G(\lambda, t)\right| \leq d(t), \\
& \Psi^{\prime}(0)=\int_{[a, b]_{\pi}} D_{\lambda} G(0, t) \Delta^{\alpha} t \\
& \quad=\int_{[a, b]_{\pi}}\left(D_{x} L\left(\sigma(t), u^{\sigma}(t), T_{\alpha}(u)(t)\right), v^{\sigma}(t)\right) \Delta^{\alpha} t \\
& \quad+\int_{[a, b]_{\pi}}\left(D_{y} L\left(\sigma(t), u^{\sigma}(t), T_{\alpha}(u)(t)\right), T_{\alpha}(v)(t)\right) \Delta^{\alpha} t .
\end{aligned}
$$

On the other hand, (106) implies that

$$
\begin{aligned}
& \left|D_{x} L\left(\sigma(t), u^{\sigma}(t), T_{\alpha}(u)(t)\right)\right| \\
& \quad \leq \widehat{a}\left(\left|u^{\sigma}(t)\right|\right)\left(\widehat{b}^{\sigma}(t)+\left|T_{\alpha}(u)(t)\right|^{p}\right) \triangleq \psi_{1}(t), \\
& \left|D_{y} L\left(\sigma(t), u^{\sigma}(t), T_{\alpha}(u)(t)\right)\right| \\
& \quad \leq \widehat{a}\left(\left|u^{\sigma}(t)\right|\right)\left(\widehat{c}^{\sigma}(t)+\left|T_{\alpha}(u)(t)\right|^{p-1}\right) \triangleq \psi_{2}(t),
\end{aligned}
$$

hence $\psi_{1} \in L_{\alpha, \Delta}^{1}\left([a, b]_{\mathbb{T}}, \mathbb{R}^{+}\right), \psi_{2} \in L_{\alpha, \Delta}^{q}\left([a, b]_{\mathbb{T}}, \mathbb{R}^{+}\right)$. Therefore, from Theorem 48, (114), and (115), there exist positive constants $C_{2}, C_{3}, C_{4}$ such that

$$
\begin{gathered}
\int_{[a, b]_{\mathbb{T}}}\left[\left(D_{x} L\left(\sigma(t), u^{\sigma}(t), T_{\alpha}(u)(t)\right), v^{\sigma}(t)\right)\right. \\
+\left(D_{y} L\left(\sigma(t), u^{\sigma}(t), T_{\alpha}(u)(t)\right), T_{\alpha}(v)\right. \\
\cdot(t))] \Delta^{\alpha} t \leq C_{2}\|v\|_{\infty}+C_{3}\left\|T_{\alpha}(v)\right\|_{L_{\Delta}^{p}} \\
\leq C_{4}\|v\|_{W_{\Delta, T}^{1, p}}
\end{gathered}
$$

and $\Phi$ has a directional derivative at $u$ and $\Phi^{\prime}(u) \in$ $\left(W_{\Delta ; a, b}^{\alpha, p}\left([a, b]_{\mathbb{T}}, \mathbb{R}^{N}\right)\right)^{*}$ given by $(108)$.

Furthermore, (106) implies that the mapping from $W_{\Delta ; a, b}^{\alpha, p}\left([a, b]_{\mathbb{T}}, \mathbb{R}^{N}\right)$ into $L_{\alpha, \Delta}^{1}\left([a, b]_{\mathbb{T}}, \mathbb{R}^{N}\right) \times L_{\alpha, \Delta}^{q}\left([a, b]_{\mathbb{T}}, \mathbb{R}^{N}\right)$ defined by

$$
u \longrightarrow\left(D_{x} L\left(\cdot, u^{\sigma}, T_{\alpha}(u)\right), D_{y} L\left(\cdot, u^{\sigma}, T_{\alpha}(u)\right)\right)
$$

is continuous, so that $\Phi^{\prime}$ is continuous from $W_{\Delta ; a, b}^{\alpha, p}\left([a, b]_{\mathbb{T}}\right.$, $\left.\mathbb{R}^{N}\right)$ into $\left(W_{\Delta ; a, b}^{\alpha, p}\left([a, b]_{\mathbb{T}}, \mathbb{R}^{N}\right)\right)^{*}$. The proof is finished.

4. An Application of the Space $W_{\Delta ; a, b}^{\alpha, p}\left([a, b]_{\mathbb{T}}, \mathbb{R}^{N}\right)$

In this section, as an application of the Sobolev's space $W_{\Delta ; a, b}^{\alpha, p}\left([a, b]_{\mathbb{T}}, \mathbb{R}^{N}\right)$. We present a recent approach via variational methods and critical point theory to obtain the existence and multiplicity of solutions for the $p$-Laplacian conformable fractional differential equations boundary value problem on time scale $\mathbb{T}$

$$
\begin{aligned}
T_{\alpha}\left(\left|T_{\alpha}(u)\right|^{p-2} T_{\alpha}(u)\right)(t) & =\nabla F(\sigma(t), u(\sigma(t))), \\
u(a)-u(b) & =0, \\
\Delta \text {-a.e. } t \in[a, b]_{\mathbb{T}}^{\kappa^{2}}, & \\
T_{\alpha}(u)(a)-T_{\alpha}(u)(b) & =0,
\end{aligned}
$$

where $T_{\alpha}(u)(t)$ denotes the conformable fractional derivative of $u$ of order $\alpha$ at $t, \sigma$ is the forward jump operator, $a, b \in$ $\mathbb{T}, 0<a<b, p>1$ and $F:[a, b]_{\mathbb{T}} \times \mathbb{R}^{N} \rightarrow \mathbb{R}$ satisfies the following assumption:

(A) $F(t, x)$ is $\Delta$-measurable in $t$ for every $x \in \mathbb{R}^{N}$ and continuously differentiable in $x$ for $\Delta$-a.e. $t \in[a, b]_{\mathbb{T}}$ and there exist $\hat{a} \in C\left(\mathbb{R}^{+}, \mathbb{R}^{+}\right), \widehat{b} \in L_{\alpha, \Delta}^{1}\left([a, b]_{\mathbb{T}}, \mathbb{R}^{+}\right)$ such that

$$
\begin{gathered}
|F(t, x)| \leq \widehat{a}(|x|) \widehat{b}(t), \\
|\nabla F(t, x)| \leq \widehat{a}(|x|) \widehat{b}(t)
\end{gathered}
$$

for all $x \in \mathbb{R}^{N}$ and $\Delta$-a.e. $t \in[a, b]_{\mathbb{T}}$, where $\nabla F(t, x)$ denotes the gradient of $F(t, x)$ in $x$.

By making a variational structure on $W_{\Delta ; a, b}^{\alpha, p}\left([a, b]_{\mathbb{T}}, \mathbb{R}^{N}\right)$, we can reduce the problem of finding solutions of (118) to the one of seeking the critical points of a corresponding functional.

Problem (118) covers the $p$-Laplacian system (for when $\mathbb{T}=\mathbb{R}, \alpha=1)$

$$
\begin{aligned}
\left(\left|u^{\prime}(t)\right|^{p-2} u^{\prime}(t)\right)^{\prime} & =\nabla F(t, u(t)), \quad \text { a.e. } t \in[a, b], \\
u(a)-u(b) & =0, \\
u^{\prime}(a)-u^{\prime}(b) & =0,
\end{aligned}
$$


and the second-order Hamiltonian system on time scale $\mathbb{T}$ (for when $p=2, \alpha=1$ )

$$
\begin{aligned}
u^{\Delta^{2}}(t) & =\nabla F(\sigma(t), u(\sigma(t))), \\
\Delta \text {-a.e. } t \in[a, b]_{\mathbb{T}}^{\kappa^{2}}, & \\
u(a)-u(b) & =0, \\
u^{\Delta}(a)-u^{\Delta}(b) & =0,
\end{aligned}
$$

as well as the second-order Hamiltonian system (for when $p=2, \alpha=1, \mathbb{T}=\mathbb{R}$ )

$$
\begin{aligned}
u^{\prime \prime}(t) & =\nabla F(t, u(t)), \quad \text { a.e. } t \in[a, b], \\
u(a)-u(b) & =0, \\
u^{\prime}(a)-u^{\prime}(b) & =0,
\end{aligned}
$$

and the second-order discrete Hamiltonian system (for when $p=2, \mathbb{T}=\mathbb{Z}, \alpha=1, b-a \geq 3$ )

$$
\begin{aligned}
\Delta^{2} u(t) & =\nabla F(t, u(t)), \quad t \in[a, b-1] \cap \mathbb{Z}, \\
u(a)-u(b) & =0, \\
\Delta u(a)-\Delta u(b) & =0 .
\end{aligned}
$$

Consider the functional $\varphi: W_{\Delta ; a, b}^{\alpha, p}\left([a, b]_{\mathbb{T}}, \mathbb{R}^{N}\right) \rightarrow \mathbb{R}$ defined by

$$
\begin{aligned}
\varphi(u)= & \frac{1}{p} \int_{[a, b]_{\mathbb{T}}}\left|T_{\alpha}(u)(t)\right|^{p} \Delta^{\alpha} t \\
& +\int_{[a, b]_{\mathbb{T}}} F\left(\sigma(t), u^{\sigma}(t)\right) \Delta^{\alpha} t .
\end{aligned}
$$

We will show the following theorems.

Theorem 53. The functional $\varphi$ is continuously differentiable on $W_{\Delta ; a, b}^{\alpha, p}\left([a, b]_{\mathbb{T}}, \mathbb{R}^{N}\right)$ and

$$
\begin{aligned}
& \left\langle\varphi^{\prime}(u), v\right\rangle \\
& =\int_{[a, b]_{\mathbb{T}}}\left|T_{\alpha}(u)\right|^{p-2}\left(T_{\alpha}(u)(t), T_{\alpha}(v)(t)\right) \Delta^{\alpha} t \\
& \quad+\int_{[a, b]_{\mathbb{T}}}\left(\nabla F\left(\sigma(t), u^{\sigma}(t)\right), v^{\sigma}(t)\right) \Delta^{\alpha} t
\end{aligned}
$$

for all $v \in W_{\Delta ; a, b}^{\alpha, p}\left([a, b]_{\mathbb{T}}, \mathbb{R}^{N}\right)$.

Proof. We choose $L(t, x, y)=(1 / p)|y|^{p}+F(t, x)$ for all $x, y \in \mathbb{R}^{N}$ and $t \in[a, b]_{\mathbb{T}}$ in Theorem 52. It is easy to get that the functional $\varphi$ is continuously differentiable on $W_{\Delta ; a, b}^{\alpha, p}\left([a, b]_{\mathbb{T}}, \mathbb{R}^{N}\right)$ and

$$
\begin{aligned}
& \left\langle\varphi^{\prime}(u), v\right\rangle \\
& =\int_{[a, b]_{\mathbb{T}}}\left|T_{\alpha}(u)\right|^{p-2}\left(T_{\alpha}(u)(t), T_{\alpha}(v)(t)\right) \Delta^{\alpha} t \\
& \quad+\int_{[a, b]_{\mathbb{T}}}\left(\nabla F\left(\sigma(t), u^{\sigma}(t)\right), v^{\sigma}(t)\right) \Delta^{\alpha} t
\end{aligned}
$$

for all $v \in W_{\Delta ; a, b}^{\alpha, p}\left([a, b]_{\mathbb{T}}, \mathbb{R}^{N}\right)$ by condition (A) and Theorem 52. The proof is complete.

Theorem 54. If $u \in W_{\Delta ; a, b}^{\alpha, p}\left([a, b]_{\mathbb{T}}, \mathbb{R}^{N}\right)$ is a critical of $\varphi$ in $W_{\Delta ; a, b}^{\alpha, p}\left([a, b]_{\mathbb{T}}, \mathbb{R}^{N}\right)$, that is, $\varphi^{\prime}(u)=0$, then $u$ is a weak solution of problem (118).

Proof. Since $\varphi^{\prime}(u)=0$, it follows from Theorem 53 that

$$
\begin{aligned}
& \int_{[a, b]_{\mathbb{T}}}\left|T_{\alpha}(u)\right|^{p-2}\left(T_{\alpha}(u)(t), T_{\alpha}(v)(t)\right) \Delta^{\alpha} t \\
& \quad+\int_{[a, b]_{\mathbb{T}}}\left(\nabla F\left(\sigma(t), u^{\sigma}(t)\right), v^{\sigma}(t)\right) \Delta^{\alpha} t=0
\end{aligned}
$$

for all $v \in W_{\Delta ; a, b}^{\alpha, p}\left([a, b]_{\mathbb{T}}, \mathbb{R}^{N}\right)$. That is

$$
\begin{array}{r}
\int_{[a, b]_{\mathbb{T}}}\left|T_{\alpha}(u)\right|^{p-2}\left(T_{\alpha}(u)(t), T_{\alpha}(v)(t)\right) \Delta^{\alpha} t \\
=-\int_{[a, b]_{\mathbb{T}}}\left(\nabla F\left(\sigma(t), u^{\sigma}(t)\right), v^{\sigma}(t)\right) \Delta^{\alpha} t
\end{array}
$$

for all $v \in W_{\Delta ; a, b}^{\alpha, p}\left([a, b]_{\mathbb{V}}, \mathbb{R}^{N}\right)$. Using condition (A) and Definition 42, we obtain that $\left|T_{\alpha}(u)\right|^{p-2} T_{\alpha}(u) \in$ $W_{\Delta ; a, b}^{\alpha, p}\left([a, b]_{\mathbb{T}}, \mathbb{R}^{N}\right)$. It follows from Theorem 45 and (74) that there exists a unique function $x \in V_{\Delta ; a, b}^{\alpha, p}\left([a, b]_{\mathbb{T}}, \mathbb{R}^{N}\right)$ such that

$$
\begin{aligned}
& x=u, \\
& T_{\alpha}\left(\left|T_{\alpha}(x)\right|^{p-2} T_{\alpha}(x)\right)(t)=\nabla F\left(\sigma(t), u^{\sigma}(t)\right) \\
& \Delta \text {-a.e. on }[a, b]_{\mathbb{T}}^{\kappa} .
\end{aligned}
$$

Equation (129) imply that

$$
\begin{aligned}
x(a)-x(b) & =0, \\
T_{\alpha}(x)(a)-T_{\alpha}(x)(b) & =0 .
\end{aligned}
$$

We identify $u \in W_{\Delta ; a, b}^{\alpha, p}\left([a, b]_{\mathbb{V}}, \mathbb{R}^{N}\right)$ with its absolutely continuous representative

$x \in V_{\Delta ; a, b}^{\alpha, p}\left([a, b]_{\mathbb{T}}, \mathbb{R}^{N}\right)$ for which (129) holds. Then, $u$ is solution of problem (118). The proof is complete.

In order to prove the existence and multiplicity of solutions for (118), we need the following definitions and theorems.

Definition 55 (see [24], $\mathrm{P}_{81}$ ). Let $X$ be a real Banach space, $I \in C^{1}(X, \mathbb{R})$ and $c \in \mathbb{R} . I$ is said to be satisfying (PS) $c^{-}$ condition on $X$ if the existence of a sequence $\left\{x_{n}\right\} \subseteq X$ such that $I\left(x_{n}\right) \rightarrow c$ and $I^{\prime}\left(x_{n}\right) \rightarrow 0$ as $n \rightarrow \infty$, implies that $c$ is a critical value of $I$.

Definition 56 (see [24], $\mathrm{P}_{81}$ ). Let $X$ be a real Banach space and $I \in C^{1}(X, \mathbb{R}) . I$ is said to be satisfying PS condition on $X$ if any sequence $\left\{x_{n}\right\} \subseteq X$ for which $I\left(x_{n}\right)$ is bounded and $I^{\prime}\left(x_{n}\right) \rightarrow 0$ as $n \rightarrow \infty$, possesses a convergent subsequence in $X$. 
Remark 57. It is clear that the PS condition implies the (PS) ${ }_{c^{-}}$ condition for each $c \in \mathbb{R}$.

Lemma 58 (Theorem 4.7, [24]). Let $X$ be a Banach space and let $\Phi \in C^{1}(X, \mathbb{R})$. Assume that $X$ splits into a direct sum of closed subspace $X=X^{-} \oplus X^{+}$with

$$
\begin{aligned}
& \operatorname{dim} X^{-}<\infty, \\
& \sup _{S_{R}^{-}} \Phi<\inf _{X^{+}} \Phi,
\end{aligned}
$$

where $S_{R}^{-}=\left\{u \in X^{-}:\|u\|=R\right\}$. Let

$$
\begin{aligned}
B_{R}^{-} & =\left\{u \in X^{-}:\|u\| \leq R\right\}, \\
M & =\left\{h \in C\left(B_{R}^{-}, X\right): h(s)=s \text { if } s \in S_{R}^{-}\right\}, \\
c & =\inf _{h \in M} \max _{s \in B_{R}^{-}} \Phi(h(s)) .
\end{aligned}
$$

Then, if $\Phi$ satisfies the $(P S)_{c}$-condition, $c$ is a critical value of $\Phi$.

As shown in [29], a deformation lemma can be proved with the weaker condition $(\mathrm{C})$ replacing the usual PS condition, and it turns out that the Saddle Point Theorem (Lemma 58) holds under (C) condition.

For the sake of convenience, in the sequel, we denote $\|\cdot\|=$ $\|\cdot\|_{W_{\Delta: a, b}^{\alpha, p}}$.
For $u \in W_{\Delta ; a, b}^{\alpha, p}\left([a, b]_{\mathbb{T}}, \mathbb{R}^{N}\right)$, let $\bar{u}=$ $\left(\int_{[a, b]_{\mathbb{T}}} 1 \Delta^{\alpha} t\right)^{-1} \int_{[a, b]_{\mathbb{T}}} u(t) \Delta t$ and $\widetilde{u}(t)=u(t)-\bar{u}$. Set $\widetilde{W}_{\Delta ; a, b}^{\alpha, p}([a$, $\left.b]_{\mathbb{T}}, \mathbb{R}^{N}\right)=\left\{u \in W_{\Delta ; a, b}^{\alpha, p}\left([a, b]_{\mathbb{T}}, \mathbb{R}^{N}\right): \int_{[a, b]_{\mathbb{T}}} u(t) \Delta^{\alpha} t=0\right\}$, then $W_{\Delta ; a, b}^{\alpha, p}\left([a, b]_{\mathbb{T}}, \mathbb{R}^{N}\right)=\widetilde{W}_{\Delta ; a, b}^{\alpha, p}\left([a, b]_{\mathbb{T}}, \mathbb{R}^{N}\right) \oplus \mathbb{R}^{N}$ and we have the following theorem.

Theorem 59. In Banach space $W_{\Delta ; a, b}^{\alpha, p}\left([a, b]_{\mathbb{T}}, \mathbb{R}^{N}\right)$, for $u \in$ $W_{\Delta ; a, b}^{\alpha, p}\left([a, b]_{\mathbb{T}}, \mathbb{R}^{N}\right),\|u\| \rightarrow \infty$ if and only if

$$
\left(|\bar{u}|^{p}+\int_{[a, b]_{\mathbb{T}}}\left|T_{\alpha}(u)(t)\right|^{p} \Delta^{\alpha} t\right)^{1 / p} \longrightarrow \infty .
$$

Proof. Firstly, we prove the necessity. Indeed, from Theorems 15 and 48 , we get

$$
\begin{gathered}
\int_{[a, b]_{\mathbb{T}}}\left|u^{\sigma}(t)\right|^{p} \Delta^{\alpha} t=\int_{[a, b]_{\mathbb{T}}}\left|\bar{u}+\widetilde{u^{\sigma}}(t)\right|^{p} \Delta^{\alpha} t \\
\leq 2^{p} \int_{[a, b]_{\mathbb{T}}}\left(|\bar{u}|^{p}+\left|\widetilde{u^{\sigma}}(t)\right|^{p}\right) \Delta^{\alpha} t \leq 2^{p} \\
\cdot \max \left\{a^{\alpha-1}, 1\right\}(b-a)\left(|\bar{u}|^{p}+\|\widetilde{u}\|_{\infty}^{p}\right) \leq 2^{p} \\
\cdot \max \left\{a^{\alpha-1}, 1\right\}(b-a) \\
\cdot\left(|\bar{u}|^{p}+K^{p} \int_{[a, b]_{\mathbb{T}}}\left|T_{\alpha}(u)(t)\right|^{p} \Delta^{\alpha} t\right) .
\end{gathered}
$$

This inequality implies

$$
\begin{aligned}
\|u\| & =\left(\int_{[a, b]_{\mathbb{T}}}\left|T_{\alpha}(u)(t)\right|^{p} \Delta^{\alpha} t+\int_{[a, b]_{\mathbb{T}}}\left|u^{\sigma}(t)\right|^{p} \Delta^{\alpha} t\right)^{1 / p} \\
& \leq\left(2^{p} \max \left\{a^{\alpha-1}, 1\right\}(b-a)\left(|\bar{u}|^{p}+K^{p} \int_{[a, b]_{\mathbb{T}}}\left|T_{\alpha}(u)(t)\right|^{p} \Delta^{\alpha} t\right)+\int_{[a, b]_{\mathbb{T}}}\left|T_{\alpha}(u)(t)\right|^{p} \Delta^{\alpha} t\right)^{1 / p} \\
& \leq\left(\left(2^{p} \max \left\{a^{\alpha-1}, 1\right\}(b-a)+K^{p}\right)+1\right)^{1 / p}\left(|\bar{u}|^{p}+\int_{[a, b]_{\mathbb{T}}}\left|T_{\alpha}(u)(t)\right|^{p} \Delta^{\alpha} t\right)^{1 / p} .
\end{aligned}
$$

That is $\|u\| \rightarrow \infty \Rightarrow\left(|\bar{u}|^{p}+\int_{[a, b]_{\mathbb{T}}}\left|T_{\alpha}(u)(t)\right|^{p} \Delta^{\alpha} t\right)^{1 / p} \rightarrow \infty$.

Secondly, we prove the sufficiency. From Theorem 15 and Hölder inequality we get

$$
\begin{aligned}
|\bar{u}| & =\left|\left(\int_{[a, b]_{\mathbb{T}}} 1 \Delta^{\alpha} t\right)^{-1} \int_{[a, b]_{\mathbb{T}}} u^{\sigma}(t) \Delta^{\alpha} t\right| \\
& \leq\left(\int_{[a, b]_{\mathbb{T}}} 1 \Delta^{\alpha} t\right)^{-1 / p}\left(\int_{[a, b]_{\mathbb{T}}}\left|u^{\sigma}(t)\right|^{p} \Delta^{\alpha} t\right)^{1 / p} .
\end{aligned}
$$

Therefore, we have

$$
\begin{aligned}
& \left(|\bar{u}|^{p}+\int_{[a, b]_{\mathbb{T}}}\left|T_{\alpha}(u)(t)\right|^{p} \Delta^{\alpha} t\right)^{1 / p} \\
& \quad \leq\left(\left(\int_{[a, b]_{\mathbb{T}}} 1 \Delta^{\alpha} t\right)^{-1}\left(\int_{[a, b]_{\mathbb{T}}}\left|u^{\sigma}(t)\right|^{p} \Delta^{\alpha} t\right)\right. \\
& \left.\quad+\int_{[a, b]_{\mathbb{T}}}\left|T_{\alpha}(u)(t)\right|^{p} \Delta^{\alpha} t\right)^{1 / p} \leq\left(\left(\int_{[a, b]_{\mathbb{T}}} 1 \Delta^{\alpha} t\right)^{-1}\right. \\
& \quad+1)^{1 / p}\|u\| .
\end{aligned}
$$


That is $\left(|\bar{u}|^{p}+\int_{[a, b]_{\top}}\left|T_{\alpha}(u)(t)\right|^{p} \Delta^{\alpha} t\right)^{1 / p} \rightarrow \infty \Rightarrow\|u\| \rightarrow \infty$. This completes the proof.

Theorem 60. Assume that (A) and the following conditions are satisfied:

$\left(F_{1}\right)$ there exist $0<\mu<p, M>0$ such that

$$
(\nabla F(t, x), x) \geq \mu F(t, x)
$$

for all $|x|>M$ and $\Delta$-a.e. $t \in[a, b]_{\mathbb{V}}$.

$\left(F_{2}\right)$ there exists $g^{\sigma} \in L_{\alpha, \Delta}^{1}\left([a, b]_{\mathbb{T}}, \mathbb{R}\right)$ such that

$$
F(t, x) \leq g(t)
$$

for all $x \in \mathbb{R}^{N}$ and $\Delta$-a.e. $t \in[a, b]_{\mathbb{T}}$.

$\left(F_{3}\right)$ there exists a subset $D$ of $[a, b]_{\mathbb{T}}$ with $\mu_{\Delta}(D)>0$ such that

$$
F(t, x) \longrightarrow-\infty \text { as }|x| \longrightarrow \infty
$$

for $\Delta$-a.e. $t \in D$.

Then problem (118) has at least one solution.

Proof. We will use the Saddle Point Theorem (Lemma 58) to prove Theorem 60. It suffices to prove that

(i) $\varphi$ satisfies (C)-condition,

(ii) $\varphi(u) \rightarrow-\infty$ as $u \in \mathbb{R}^{N},\|u\| \rightarrow \infty$,

(iii) $\varphi(u) \rightarrow+\infty$ as $u \in \widetilde{W}_{\Delta ; a, b}^{\alpha, p}\left([a, b]_{\mathbb{V}}, \mathbb{R}^{N}\right),\|u\| \rightarrow \infty$.

Firstly, we prove (i).

Let $\left\{u_{n}\right\} \subset W_{\Delta ; a, b}^{\alpha, p}\left([a, b]_{\mathbb{T}}, \mathbb{R}^{N}\right)$ be (C)-sequence of $\varphi$, that is $\varphi\left(u_{n}\right)$ is bounded and $\left(1+\left\|u_{n}\right\|\right) \varphi^{\prime}\left(u_{n}\right) \rightarrow 0$ as $n \rightarrow \infty$. Then there exists a positive constant $C_{5}$ such that

$$
\begin{aligned}
\left|\varphi\left(u_{n}\right)\right| & \leq C_{5}, \\
\left(1+\left\|u_{n}\right\|\right)\left\|\varphi^{\prime}\left(u_{n}\right)\right\| & \leq C_{5}
\end{aligned}
$$

for all $n \in \mathbb{N}$. In accordance with (A), $\left(F_{1}\right),(124)$, and (125), we have

$$
\begin{aligned}
C_{5} & +p C_{5} \geq\left(1+\left\|u_{n}\right\|\right)\left\|\varphi^{\prime}\left(u_{n}\right)\right\|-p \varphi\left(u_{n}\right) \\
& \geq\left\langle\varphi^{\prime}\left(u_{n}\right), u_{n}\right\rangle-p \varphi\left(u_{n}\right) \\
& =\int_{[a, b]_{\pi}}\left[\left(\nabla F\left(\sigma(t), u_{n}^{\sigma}(t)\right), u_{n}^{\sigma}(t)\right)\right.
\end{aligned}
$$

$$
\begin{aligned}
& \left.-p F\left(\sigma(t), u_{n}^{\sigma}(t)\right)\right] \Delta^{\alpha} t \\
& =\int_{\left\{u_{n}^{\sigma}(t)>M\right\}}\left[\left(\nabla F\left(\sigma(t), u_{n}^{\sigma}(t)\right), u_{n}^{\sigma}(t)\right)\right. \\
& \left.-p F\left(\sigma(t), u_{n}^{\sigma}(t)\right)\right] \Delta^{\alpha} t \\
& +\int_{\left\{u_{n}^{\sigma}(t) \leq M\right\}}\left[\left(\nabla F\left(\sigma(t), u_{n}^{\sigma}(t)\right), u_{n}^{\sigma}(t)\right)\right. \\
& \left.-p F\left(\sigma(t), u_{n}^{\sigma}(t)\right)\right] \Delta^{\alpha} t \geq(\mu-p) \\
& \cdot \int_{\left\{u_{n}^{\sigma}(t)>M\right\}} F\left(\sigma(t), u_{n}^{\sigma}(t)\right) \Delta^{\alpha} t-(p+M) \\
& \cdot \max _{|x| \leq M} \widehat{a}(|x|) \int_{[a, b]_{\top}} \widehat{b}^{\sigma}(t) \Delta^{\alpha} t
\end{aligned}
$$

for all $n \in \mathbb{N}$. This inequality gives that there exists a constant $\widetilde{C}_{6}$ such that

$$
\int_{\left\{u_{n}^{\sigma}(t)>M\right\}} F\left(\sigma(t), u_{n}^{\sigma}(t)\right) \Delta^{\alpha} t \geq \widetilde{C}_{6}
$$

for all $n \in \mathbb{N}$. From condition (A), we have

$$
\begin{aligned}
& \int_{\left\{u_{n}^{\sigma}(t) \leq M\right\}} F\left(\sigma(t), u_{n}^{\sigma}(t)\right) \Delta^{\alpha} t \\
& \geq-\int_{\left\{u_{n}^{\sigma}(t) \leq M\right\}} \widehat{a}\left(\left|u_{n}^{\sigma}(t)\right|\right) \widehat{b}^{\sigma}(t) \Delta^{\alpha} t \\
& \quad \geq-\max _{|x| \leq M} \widehat{a}(|x|) \int_{[a, b]_{\pi}} \widehat{b}^{\sigma}(t) \Delta^{\alpha} t .
\end{aligned}
$$

Combining (143) with (144), one has

$$
\begin{aligned}
& \int_{[a, b]_{\mathbb{T}}} F\left(\sigma(t), u_{n}^{\sigma}(t)\right) \Delta^{\alpha} t \\
& =\int_{\left\{u_{n}^{\sigma}(t)>M\right\}} F\left(\sigma(t), u_{n}^{\sigma}(t)\right) \Delta^{\alpha} t \\
& \quad+\int_{\left\{u_{n}^{\sigma}(t) \leq M\right\}} F\left(\sigma(t), u_{n}^{\sigma}(t)\right) \Delta^{\alpha} t \geq C_{6},
\end{aligned}
$$

where $C_{6}=\widetilde{C}_{6}-\max _{|x| \leq M} \widehat{a}(|x|) \int_{[a, b]_{T}} \widehat{b}^{\sigma}(t) \Delta^{\alpha} t$. Moreover, by (124), (141), and (145), one has

$$
C_{5} \geq \varphi\left(u_{n}\right) \geq \frac{1}{p} \int_{[a, b]_{T}}\left|T_{\alpha}\left(u_{n}\right)(t)\right|^{p} \Delta^{\alpha} t+C_{6}
$$


for all $n \in \mathbb{N}$. This shows that there exists constant $C_{7}$ such that

$$
\int_{[a, b]_{\mathbb{T}}}\left|T_{\alpha}\left(u_{n}\right)(t)\right|^{p} \Delta^{\alpha} t \leq C_{7}
$$

for all $n \in \mathbb{N}$. It follows from Theorem 48 and (147) that there exists constant $C_{8}$ such that

$$
\left\|\tilde{u}_{n}\right\|_{\infty} \leq C_{8}
$$

for all $n \in \mathbb{N}$.

Now, we will assert the sequence $\left\{\bar{u}_{n}\right\}$ is bounded. If not, we may assume, without loss of generality, that $\left|\bar{u}_{n}\right| \rightarrow \infty$ as $n \rightarrow \infty$. Set $v_{n}=u_{n} /\left\|u_{n}\right\|=\bar{u}_{n} /\left\|u_{n}\right\|+\widetilde{u}_{n} /\left\|u_{n}\right\|=\bar{v}_{n}+\widetilde{v}_{n}$, then $\left\{v_{n}\right\}$ is bounded in $W_{\Delta ; a, b}^{\alpha, p}\left([a, b]_{\mathbb{T}}, \mathbb{R}^{N}\right)$. By Remark 51, there is a subsequence of $\left\{v_{n}\right\}$ (for simplicity denoted again by $\left\{v_{n}\right\}$ ) such that

$$
\begin{aligned}
& v_{n} \rightarrow v \quad \text { in } W_{\Delta ; a, b}^{\alpha, p}\left([a, b]_{\mathbb{T}}, \mathbb{R}^{N}\right), \\
& v_{n} \longrightarrow v \quad \text { in } C\left([a, b]_{\mathbb{T}}, \mathbb{R}^{N}\right) .
\end{aligned}
$$

Combining with (148), $\left\{\tilde{u}_{n}\right\}$ is bounded in $C\left([a, b]_{\mathbb{T}}, \mathbb{R}^{N}\right)$, hence, $v \in \mathbb{R}^{N}, v \neq 0$. So $\left|u_{n}(t)\right| \rightarrow \infty$ as $n \rightarrow \infty$ for all $t \in[a, b]_{\mathbb{T}}$. By $\left(F_{3}\right)$, we have

$$
\begin{array}{r}
\limsup _{n \rightarrow \infty} \int_{[a, b]_{\mathbb{T}}} F\left(\sigma(t), u_{n}^{\sigma}(t)\right) \Delta^{\alpha} t \\
\leq \limsup _{n \rightarrow \infty} \int_{D} F\left(\sigma(t), u_{n}^{\sigma}(t)\right) \Delta^{\alpha} t \\
\quad+\int_{[a, b]_{\mathbb{T}}}\left|g^{\sigma}(t)\right| \Delta^{\alpha} t \longrightarrow-\infty,
\end{array}
$$

which contradicts (145).

Consequently, by Theorem 59, $\left\{u_{n}\right\}$ is bounded in $W_{\Delta ; a, b}^{\alpha, p}\left([a, b]_{\mathbb{T}}, \mathbb{R}^{N}\right)$. Again, from Remark 51 , there is a subsequence of $\left\{u_{n}\right\}$ (for simplicity denoted again by $\left\{u_{n}\right\}$ ) such that

$$
\begin{aligned}
& u_{n} \rightarrow u \quad \text { in } W_{\Delta ; a, b}^{\alpha, p}\left([a, b]_{\mathbb{T}}, \mathbb{R}^{N}\right), \\
& u_{n} \longrightarrow u \quad \text { in } C\left([a, b]_{\mathbb{I}}, \mathbb{R}^{N}\right) .
\end{aligned}
$$

According to (152), $\left\{u_{n}\right\}$ is bounded in $C\left([a, b]_{\mathbb{T}}, \mathbb{R}^{N}\right)$, then from condition (A), there exists positive constant $C_{9}$ such that

$$
\begin{aligned}
& \left|\int_{[a, b]_{\mathbb{T}}}\left(\nabla F\left(\sigma(t), u_{n}^{\sigma}(t)\right), u^{\sigma}(t)-u_{n}^{\sigma}(t)\right) \Delta^{\alpha} t\right| \\
& \quad \leq \int_{[a, b]_{\mathbb{T}}}\left|\nabla F\left(\sigma(t), u_{n}^{\sigma}(t)\right)\right|\left|u^{\sigma}(t)-u_{n}^{\sigma}(t)\right| \Delta^{\alpha} t \\
& \quad \leq C_{9} \int_{[a, b]_{\mathbb{T}}} \widehat{b}^{\sigma}(t)\left|u^{\sigma}(t)-u_{n}^{\sigma}(t)\right| \Delta^{\alpha} t \\
& \quad \leq C_{9}\left\|u-u_{n}\right\|_{\infty} \int_{[a, b]_{\mathbb{T}}} \widehat{b}^{\sigma}(t) \Delta^{\alpha} t .
\end{aligned}
$$

Combining with (152), we get

$$
\begin{aligned}
\int_{[a, b]_{\mathbb{T}}}\left(\nabla F\left(\sigma(t), u_{n}^{\sigma}(t)\right), u^{\sigma}(t)-u_{n}^{\sigma}(t)\right) \Delta^{\alpha} t & \longrightarrow 0 \\
\text { as } n & \longrightarrow \infty .
\end{aligned}
$$

Be aware of

$$
\begin{aligned}
& \left\langle\varphi^{\prime}\left(u_{n}\right), u-u_{n}\right\rangle=\int_{[a, b]_{\mathbb{T}}}\left|T_{\alpha}\left(u_{n}\right)(t)\right|^{p-2} \\
& \cdot\left(T_{\alpha}\left(u_{n}\right)(t), T_{\alpha}(u)(t)-T_{\alpha}\left(u_{n}\right)(t)\right) \Delta^{\alpha} t \\
& \quad+\int_{[a, b]_{\mathbb{T}}}\left[\nabla F\left(\sigma(t), u_{n}^{\sigma}(t)\right), u^{\sigma}(t)\right. \\
& \left.\quad-u_{n}^{\sigma}(t)\right] \Delta^{\alpha} t, \\
& \left\langle\varphi^{\prime}\left(u_{n}\right), u-u_{n}\right\rangle \rightarrow 0 \text { as } n \rightarrow \infty ;
\end{aligned}
$$

one has

$$
\begin{aligned}
\int_{[a, b]_{\mathbb{T}}}\left|T_{\alpha}\left(u_{n}\right)(t)\right|^{p-2} & \\
\cdot\left(T_{\alpha}\left(u_{n}\right)(t), T_{\alpha}(u)(t)-T_{\alpha}\left(u_{n}\right)(t)\right) \Delta^{\alpha} t & \longrightarrow 0 \\
\text { as } n & \longrightarrow \infty .
\end{aligned}
$$

Moreover, it is easy to derive from (152) that

$$
\begin{aligned}
\int_{[a, b]_{\mathbb{T}}}\left|u_{n}^{\sigma}(t)\right|^{p-2}\left(u_{n}^{\sigma}(t), u^{\sigma}(t)-u_{n}^{\sigma}(t)\right) \Delta^{\alpha} t & \longrightarrow 0 \\
\text { as } n & \longrightarrow \infty .
\end{aligned}
$$

Define

$$
\begin{gathered}
\phi(u)=\frac{1}{p}\|u\|^{p}=\frac{1}{p}\left(\int_{[a, b]_{\mathbb{T}}}\left|u^{\sigma}(t)\right|^{p} \Delta^{\alpha} t\right. \\
\left.+\int_{[a, b]_{\mathbb{T}}}\left|T_{\alpha}(u)(t)\right|^{p} \Delta^{\alpha} t\right) .
\end{gathered}
$$

Then one has

$$
\begin{aligned}
& \left\langle\phi^{\prime}\left(u_{n}\right), u-u_{n}\right\rangle=\int_{[a, b]_{\mathbb{T}}}\left|T_{\alpha}\left(u_{n}\right)(t)\right|^{p-2} \\
& \cdot\left(T_{\alpha}\left(u_{n}\right)(t), T_{\alpha}(u)(t)-T_{\alpha}\left(u_{n}\right)(t)\right) \Delta^{\alpha} t \\
& \quad+\int_{[a, b]_{\mathbb{T}}}\left|u_{n}^{\sigma}(t)\right|^{p-2}\left(u_{n}^{\sigma}(t), u^{\sigma}(t)-u_{n}^{\sigma}(t)\right) \Delta^{\alpha} t \\
& \quad \longrightarrow 0
\end{aligned}
$$

as $n \rightarrow \infty$. By Hölder inequality, we get

$$
\begin{aligned}
0 & \leq\left(\left\|u_{n}\right\|-\|u\|\right)\left(\left\|u_{n}\right\|^{p-1}-\|u\|^{p-1}\right) \\
& \leq\left\langle\phi^{\prime}\left(u_{n}\right)-\phi^{\prime}(u), u_{n}-u\right\rangle .
\end{aligned}
$$

This combined with (159) yields $\left\|u_{n}\right\| \rightarrow\|u\|$. It is easy to derive from (151) and Theorem 47 that $u_{n} \rightarrow u$ in $W_{\Delta ; a, b}^{\alpha, p}\left([a, b]_{\mathbb{T}}, \mathbb{R}^{N}\right)$. 
Secondly, we prove (ii).

For any $u \in \mathbb{R}^{N}$, combining (124) with $\left(F_{2}\right)$ and $\left(F_{3}\right)$, we have

$$
\begin{aligned}
\varphi(u) & =\int_{[a, b]_{\top}} F\left(\sigma(t), u^{\sigma}(t)\right) \Delta^{\alpha} t \\
& \leq \int_{D} F\left(\sigma(t), u^{\sigma}(t)\right) \Delta^{\alpha} t+\int_{[a, b]_{\top} \backslash D} g^{\sigma}(t) \Delta^{\alpha} t \\
& \leq \int_{D} F\left(\sigma(t), u^{\sigma}(t)\right) \Delta^{\alpha} t+\int_{[a, b]_{\top}}\left|g^{\sigma}(t)\right| \Delta^{\alpha} t \\
& \longrightarrow-\infty
\end{aligned}
$$

as $n \rightarrow \infty$. This shows (ii) holds.

Thirdly, we prove (iii).

For $s \in \mathbb{R},|x| \geq M$ and $\Delta$-a.e. $t \in[a, b]_{\mathbb{T}}$, we set

$$
\begin{aligned}
& G(s)=F(t, s x), \\
& H(s)=G^{\prime}(s)-\frac{\mu}{s} G(s) .
\end{aligned}
$$

Make use of $\left(F_{1}\right)$, when $s \geq M /|x|$,

$$
H(s)=\frac{1}{s}((\nabla F(t, s x), s x)-\mu F(t, s x)) \geq 0
$$

holds. In addition, using (162), $G(s)$ satisfies

$$
G^{\prime}(s)=H(s)+\frac{\mu}{s} G(s) .
$$

So, when $s \geq M /|x|$,

$$
F(t, s x)=s^{\mu}\left(F(t, x)+\int_{1}^{s} \tau^{-\mu} H(\tau) d \tau\right) .
$$

Besides, depending on condition (A) and (163), for $|x| \geq M$ and $\Delta$-a.e. $t \in[a, b]_{\mathbb{T}}$, we assert

$$
\left(\frac{M}{|x|}\right)^{\mu} F(t, x) \geq F\left(t, x \frac{M}{|x|}\right) \geq-a_{0} \widehat{b}(t),
$$

where $a_{0}=\max _{|x| \leq M} \widehat{a}(|x|)$. This implies that

$$
F(t, x) \geq-a_{0} \widehat{b}(t)\left(\left(\frac{|x|}{M}\right)^{\mu}+1\right)
$$

for all $x \in \mathbb{R}^{N}$ and $\Delta$-a.e. $t \in[a, b]_{\mathbb{V}}$. Moreover, by virtue of Theorem 48 and (124), we obtain

$$
\begin{aligned}
& \varphi(u) \\
& \geq \frac{1}{p} \int_{[a, b]_{\top}}\left|T_{\alpha}(u)(t)\right|^{p} \Delta^{\alpha} t \\
& -\left(\frac{a_{0}}{M^{\mu}}\right)\|u\|_{\infty}^{\mu} \int_{[a, b]_{\top}} \hat{b}^{\sigma}(t) \Delta^{\alpha} t \\
& -a_{0} \int_{[a, b]_{\top}} \hat{b}^{\sigma}(t) \Delta^{\alpha} t \\
& =\frac{1}{2 p} \int_{[a, b]_{\top}}\left|T_{\alpha}(u)(t)\right|^{p} \Delta^{\alpha} t \\
& +\frac{1}{2 p} \int_{[a, b]_{T}}\left|T_{\alpha}(u)(t)\right|^{p} \Delta^{\alpha} t \\
& -\left(\frac{a_{0}}{M^{\mu}}\right)\|u\|_{\infty}^{\mu} \int_{[a, b]_{\top}} \hat{b}^{\sigma}(t) \Delta^{\alpha} t \\
& -a_{0} \int_{[a, b]_{\top}} \widehat{b}^{\sigma}(t) \Delta^{\alpha} t \\
& \geq \frac{1}{2 p} \int_{[a, b]_{\top}}\left|T_{\alpha}(u)(t)\right|^{p} \Delta^{\alpha} t+\frac{1}{2 p} K^{-p}\|u\|_{\infty}^{p} \\
& -\left(\frac{a_{0}}{M^{\mu}}\right)\|u\|_{\infty}^{\mu} \int_{[a, b]_{\top}} \widehat{b}^{\sigma}(t) \Delta^{\alpha} t \\
& -a_{0} \int_{[a, b]_{\pi}} \widehat{b}^{\sigma}(t) \Delta^{\alpha} t \\
& \geq \frac{1}{2 p} \int_{[a, b]_{\top}}\left|T_{\alpha}(u)(t)\right|^{p} \Delta^{\alpha} t \\
& +\frac{1}{2 p} K^{-p}\left(\max \left\{a^{\alpha-1}, 1\right\}\right)^{-1} \int_{[a, b]_{\mathrm{T}}}\left|u^{\sigma}(t)\right|^{p} \Delta^{\alpha} t \\
& -\left(\frac{a_{0} K^{p}}{M^{\mu}}\right)\|u\|^{\mu} \int_{[a, b]_{\top}} \widehat{b}^{\sigma}(t) \Delta^{\alpha} t \\
& -a_{0} \int_{[a, b]_{\pi}} \hat{b}^{\sigma}(t) \Delta^{\alpha} t \\
& \geq \min \left\{\frac{1}{2 p}, \frac{1}{2 p} K^{-p}\left(\max \left\{a^{\alpha-1}, 1\right\}\right)^{-1}\right\}\|u\|^{p} \\
& -\left(\frac{a_{0} K^{p}}{M^{\mu}}\right)\|u\|^{\mu} \int_{[a, b]_{\top}} \widehat{b}^{\sigma}(t) \Delta^{\alpha} t \\
& -a_{0} \int_{[a, b]_{\top}} \widehat{b}^{\sigma}(t) \Delta^{\alpha} t
\end{aligned}
$$

for all $u \in \widetilde{W}_{\Delta, T}^{1, p}\left([a, b]_{\mathbb{T}}, \mathbb{R}^{N}\right)$. Since $0<\mu<p$, (iii) holds.

It follows from Lemma 58 and Theorem 54 that Theorem 60 holds.

Example 61. Let $\mathbb{T}=\bigcup_{k=0}^{\infty}[2 k, 2 k+1], \alpha=1 / 2, a=$ $1, b=2015, N=5$. Consider the 4-Laplacian conformable 
fractional differential equation boundary value problem on time scale T

$$
\begin{aligned}
T_{1 / 2}\left(\left|T_{1 / 2}(u)\right|^{2} T_{1 / 2}(u)\right)(t)= & \nabla F(\sigma(t), u(\sigma(t))), \\
& \Delta \text {-a.e. } t \in[1,2015]_{\mathbb{T}}^{\kappa^{2}}, \\
u(1)-u(2015)= & 0, \\
T_{\alpha}(u)(1)-T_{\alpha}(u)(2015)= & 0,
\end{aligned}
$$

where $F(t, x)=-|x|^{2}-|((1,1,2,2,3), x)|$.

In virtue of $F(t, x)=-|x|^{2}-|((1,1,2,2,3), x)|, \nabla F(t, x)=$ $-2 x-(((1,1,2,2,3), x) /|((1,1,2,2,3), x)|)(1,1,2,2,3)$, all conditions of Theorem 60 hold with $p=4, \mu=3, M=$ $1, g(t) \equiv 0$. It is easy to derive from Theorem 60 that problem (169) has at least one solution. Moreover, 0 is not the solution of problem (169). Thus, problem (169) has at least one nontrivial solution. lary.

According to Theorem 60, we have the following corol-

Corollary 62. Assume that $(A),\left(F_{1}\right)$, and the following condition are satisfied.

$\left(F_{4}\right) F(t, x) \rightarrow-\infty$ as $|x| \rightarrow \infty$ uniformly for $\Delta$-a.e. $t \in$ $[a, b]_{\mathbb{T}}$.

Then problem (118) has at least one solution.

Theorem 63. Suppose that $(A),\left(F_{1}\right)$ and the following condition are satisfied:

$\left(F_{5}\right) \int_{[a, b]_{\pi}} F(\sigma(t), x) \Delta^{\alpha} t \rightarrow-\infty$ as $|x| \rightarrow \infty$.

$\left(F_{6}\right)$ Assume that $-F(t, \cdot)$ is $(\beta, \gamma)$-subconvex with $\gamma>0 \Delta$ a.e. $t \in[a, b]_{\mathbb{T}}$, that is,

$$
F(t, \beta(x+y)) \geq \gamma(F(t, x)+F(t, y))
$$

$$
\text { for all } x, y \in \mathbb{R}^{N} \text { and } \Delta \text {-a.e. } t \in[a, b]_{\mathbb{T}} \text {. }
$$

Then problem (118) has at least one solution.

Proof. It follows directly from $\left(F_{5}\right)$ that

$$
\varphi(u) \longrightarrow-\infty \quad \text { as } u \in \mathbb{R}^{N},\|u\| \longrightarrow \infty
$$

Analogous to the proof of Theorem 60, we can obtain

$$
\begin{aligned}
& \varphi(u) \longrightarrow+\infty \\
& \quad \text { as } u \in \widetilde{W}_{\Delta ; a, b}^{\alpha, p}\left([a, b]_{\mathbb{T}}, \mathbb{R}^{N}\right),\|u\| \longrightarrow \infty .
\end{aligned}
$$

Next, we will verify $\varphi$ satisfies (C)-condition. Let $\left\{u_{n}\right\} \subset$ $W_{\Delta ; a, b}^{\alpha, p}\left([a, b]_{\mathbb{T}}, \mathbb{R}^{N}\right)$ be a $(\mathrm{C})$-sequence; that is, $\varphi\left(u_{n}\right)$ is bounded and $\left(1+\left\|u_{n}\right\|\right) \varphi^{\prime}\left(u_{n}\right) \rightarrow 0$ as $n \rightarrow \infty$. Using the same method as that of (145), (147), and (148) in the proof of (i) in Theorem 60, there exist constants $C_{10}, C_{11}$ and $C_{12}$ such that

$$
\begin{array}{r}
\int_{[a, b]_{\mathbb{T}}} F\left(\sigma(t), u_{n}^{\sigma}(t)\right) \Delta^{\alpha} t \geq C_{10}, \\
\int_{[a, b]_{\mathbb{T}}}\left|T_{\alpha}\left(u_{n}\right)(t)\right|^{p} \Delta^{\alpha} t \leq C_{11}, \\
\left\|\tilde{u}_{n}\right\|_{\infty} \leq C_{12}
\end{array}
$$

for all $n \in \mathbb{N}$. By means of $\left(F_{6}\right)$, we obtain

$$
\begin{aligned}
C_{10} \leq & \int_{[a, b]_{\mathbb{T}}} F\left(\sigma(t), u_{n}^{\sigma}(t)\right) \Delta^{\alpha} t \\
\leq & \frac{1}{\gamma} \int_{[a, b]_{\mathbb{T}}} F\left(\sigma(t), \beta \bar{u}_{n}\right) \Delta^{\alpha} t \\
& -\int_{[a, b]_{\mathbb{T}}} F\left(\sigma(t),-\widetilde{u}_{n}^{\sigma}(t)\right) \Delta^{\alpha} t \\
\leq & \frac{1}{\gamma} \int_{[a, b]_{\mathbb{T}}} F\left(\sigma(t), \beta \bar{u}_{n}\right) \Delta^{\alpha} t \\
& -\max _{|x| \leq C_{12}} \hat{a}(|x|) \int_{[a, b]_{\mathbb{T}}} \widehat{b}^{\sigma}(t) \Delta^{\alpha} t
\end{aligned}
$$

for all $n \in \mathbb{N}$, this conclusion shows that $\left\{\bar{u}_{n}\right\}$ is bounded. Combining with (174), we are sure $\left\{u_{n}\right\}$ is bounded. In a way similar to the proof of (i) in Theorem 60 we can prove that $\left\{u_{n}\right\}$ has a convergent subsequence, thus, $\varphi$ satisfies condition (C).

It follows from Lemma 58 and Theorem 54 that Theorem 63 holds.

Example 64. Let $\mathbb{T}=\mathbb{R}, \alpha=1, a=\pi, b=2 \pi, N=$ 3 . Consider the 5 -Laplacian differential equation boundary value problem

$$
\begin{aligned}
\left(\left|u^{\prime}(t)\right|^{3} u^{\prime}(t)\right)^{\prime} & =\nabla F(t, u(t)), \quad \text { a.e. } t \in[\pi, 2 \pi], \\
u(\pi)-u(2 \pi) & =0, \\
u^{\prime}(\pi)-u^{\prime}(2 \pi) & =0,
\end{aligned}
$$

where $F(t, x)=-|x|^{3}-|((2014,2015,2016), x)|$.

In virtue of $F(t, x)=-|x|^{3}-\mid((2014,2015,2016)$, $x) \mid$, and $\nabla F(t, x)=-3|x| x-(((2014,2015,2016), x) /$ $|((2014,2015,2016), x)|)(2014,2015,2016)$, all conditions of Theorem 63 hold $p=5, \mu=4, M=2$. It follows directly from Theorem 63 that problem (176) has at least one solution. Furthermore, 0 is not the solution of problem (176). Therefore, problem (176) has at least one nontrivial solution.

\section{Competing Interests}

The authors declare that they have no competing interests.

\section{Acknowledgments}

This work is supported by the National Natural Science Foundation of China under Grants 11361072 and 11561072 and 
the Natural Sciences Foundation of Yunnan Province under Grant 2016FB011.

\section{References}

[1] S. Hilger, "Analysis on measure chains-a unified approach to continuous and discrete calculus," Results in Mathematics, vol. 18, no. 1-2, pp. 18-56, 1990.

[2] S. Hilger, "Differential and difference calculus Unified!," Nonlinear Analysis: Theory, Methods \& Applications, vol. 30, no. 5, pp. 2683-2694, 1997.

[3] G. Nordlund, "Time-scales in futures research and forecasting," Futures, vol. 44, no. 4, pp. 408-414, 2012.

[4] Q. Sheng, M. Fadag, J. Henderson, and J. M. Davis, "An exploration of combined dynamic derivatives on time scales and their applications," Nonlinear Analysis. Real World Applications. An International Multidisciplinary Journal, vol. 7, no. 3, pp. 395413, 2006.

[5] H. T. Zhang and Y. K. Li, "Almost periodic solutions to dynamic equations on time scales," Journal of the Egyptian Mathematical Society, vol. 21, no. 1, pp. 3-10, 2013.

[6] X.-H. Deng, Q.-R. Wang, and Z. Zhou, "Oscillation criteria for second order nonlinear delay dynamic equations on time scales," Applied Mathematics and Computation, vol. 269, pp. 834-840, 2015.

[7] S. H. Hong and Y. Z. Peng, "Almost periodicity of set-valued functions and set dynamic equations on time scales," Information Sciences, vol. 330, pp. 157-174, 2016.

[8] S.-Y. Zhang, Q.-R. Wang, and Q. Kong, "Asymptotics and oscillation of $n$ th-order nonlinear dynamic equations on time scales," Applied Mathematics and Computation, vol. 275, pp. 324-334, 2016.

[9] J. W. Zhou and Y. K. Li, "Variational approach to a class of p-Laplacian systems on time scales," Advances in Difference Equations, vol. 2013, article 297, 2013.

[10] Y. K. Li and J. W. Zhou, "Existence of solutions for a class of damped vibration problems on time scales," Advances in Difference Equations, vol. 2010, Article ID 727486, 27 pages, 2010.

[11] P. A. Williams, "Fractional calculus on time scales with Taylor's theorem," Fractional Calculus and Applied Analysis, vol. 15, no. 4, pp. 616-638, 2012.

[12] N. Benkhettou, A. Hammoudi, and D. F. M. Torres, "Existence and uniqueness of solution for a fractional Riemann-Liouville initial value problem on time scales," Journal of King Saud University-Science, vol. 28, no. 1, pp. 87-92, 2016.

[13] R. Khalil, M. Al Horani, A. Yousef, and M. Sababheh, "A new definition of fractional derivative," Journal of Computational and Applied Mathematics, vol. 264, pp. 65-70, 2014.

[14] N. Benkhettou, S. Hassani, and D. F. M. Torres, "A conformable fractional calculus on arbitrary time scales," Journal of King Saud University-Science, vol. 28, no. 1, pp. 93-98, 2016.

[15] R. P. Agarwal, M. Benchohra, and S. Hamani, "A survey on existence results for boundary value problems of nonlinear fractional differential equations and inclusions," Acta Applicandae Mathematicae, vol. 109, no. 3, pp. 973-1033, 2010.

[16] D. A. Benson, S. W. Wheatcraft, and M. M. Meerschaert, "Application of a fractional advection-dispersion equation," Water Resources Research, vol. 36, no. 6, pp. 1403-1412, 2000.
[17] V. Lakshmikantham and A. S. Vatsala, "Basic theory of fractional differential equations," Nonlinear Analysis. Theory, Methods \& Applications. An International Multidisciplinary Journal. Series A: Theory and Methods, vol. 69, no. 8, pp. 2677-2682, 2008.

[18] G. H. Salgado and L. A. Aguirre, "A hybrid algorithm for Caputo fractional differential equations," Communications in Nonlinear Science and Numerical Simulation, vol. 33, pp. 133-140, 2016.

[19] S. Q. Zhang, "Existence of a solution for the fractional differential equation with nonlinear boundary conditions," Computers \& Mathematics with Applications, vol. 61, no. 4, pp. 1202-1208, 2011.

[20] K. H. Zhao and P. Gong, "Positive solutions of Riemann-Stieltjes integral boundary problems for the nonlinear coupling system involving fractional-order differential," Advances in Difference Equations, vol. 2014, article 254, 18 pages, 2014.

[21] W. H. Jiang, "The existence of solutions for boundary value problems of fractional differential equations at resonance," Nonlinear Analysis. Theory, Methods \& Applications. An International Multidisciplinary Journal. Series A: Theory and Methods, vol. 74, no. 5, pp. 1987-1994, 2011.

[22] F. Jiao and Y. Zhou, "Existence of solutions for a class of fractional boundary value problems via critical point theory," Computers \& Mathematics with Applications, vol. 62, no. 3, pp. 1181-1199, 2011.

[23] P. H. Rabinowitz, "Minimax method in critical point theory with applications to differential equations," in Proceedings of the CBMS Regional Conference Series in Mathematics, vol. 65 of American Mathematical Society, Providence, RI, USA, 1986.

[24] J. Mawhin and M. Willem, Critical Point Theory and Hamiltonian Systems, vol. 74 of Applied Mathematical Sciences, Springer, New York, NY, USA, 1989.

[25] J. W. Zhou and Y. K. Li, “Sobolev's spaces on time scales and its application to a class of second order Hamiltonian systems on time scales," Nonlinear Analysis, vol. 73, pp. 1375-1388, 2010.

[26] F. Jiao and Y. Zhou, "Existence results for fractional boundary value problem via critical point theory," International Journal of Bifurcation and Chaos in Applied Sciences and Engineering, vol. 22, no. 4, Article ID 1250086, 2012.

[27] G. S. Guseinov, "Integration on time scales," Journal of Mathematical Analysis and Applications, vol. 285, no. 1, pp. 107-127, 2003.

[28] R. P. Agarwal, V. Otero-Espinar, K. Perera, and D. R. Vivero, "Basic properties of Sobolev's spaces on time scales," Advances in Difference Equations, vol. 2006, Article ID 38121, 14 pages, 2006.

[29] C. K. Zhong, X. L. Fan, and W. Y. Chen, Introduction to Nonlinear Functional Analysis, Lanzhou University Press, Lanzhou, China, 2004 (Chinese). 


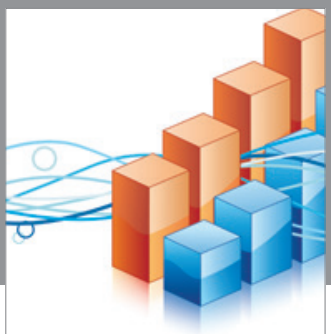

Advances in

Operations Research

vatem alat4

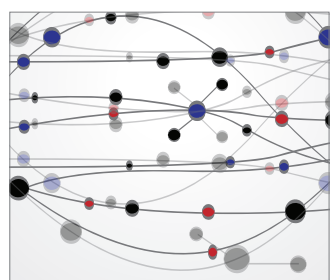

\section{The Scientific} World Journal
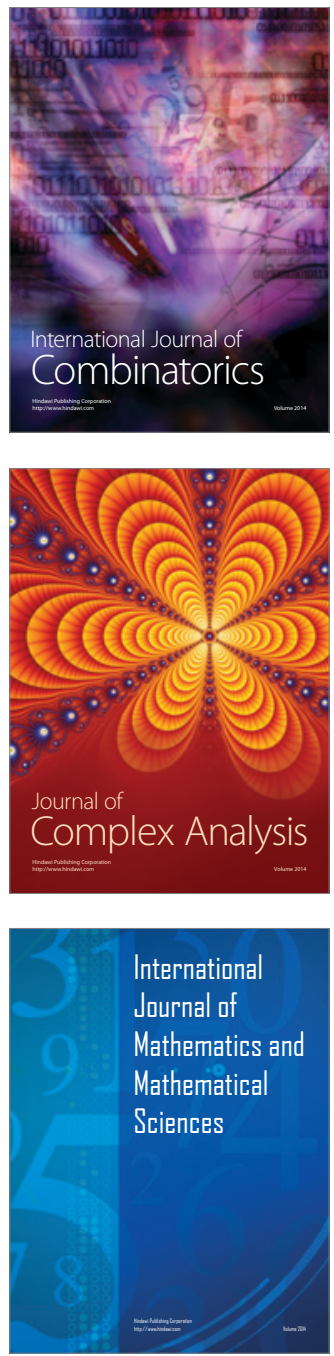
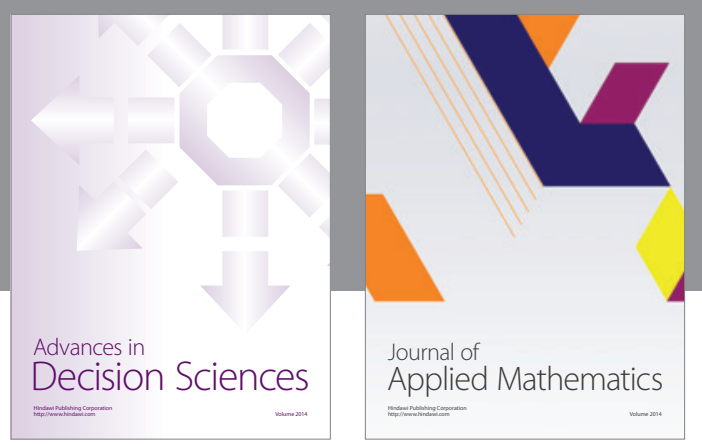

Algebra

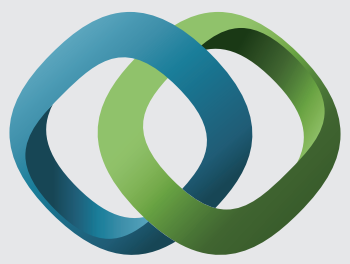

\section{Hindawi}

Submit your manuscripts at

http://www.hindawi.com
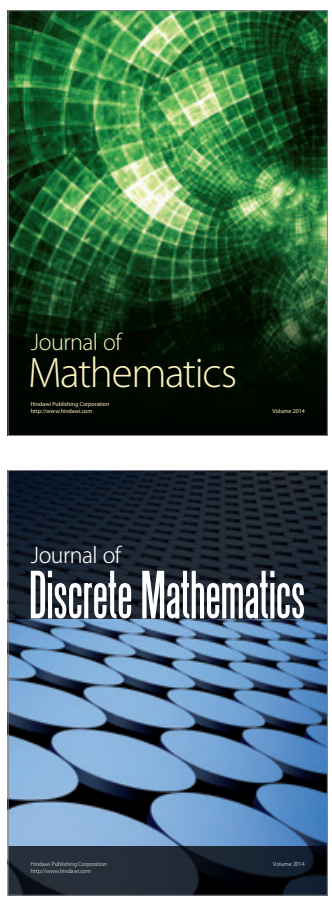

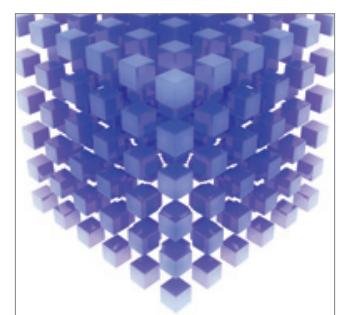

Mathematical Problems in Engineering
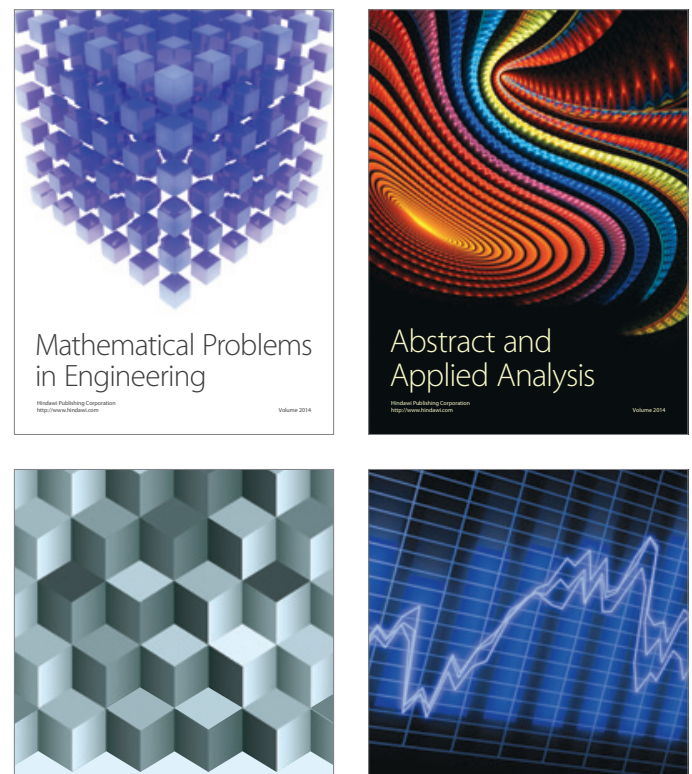

Journal of

Function Spaces

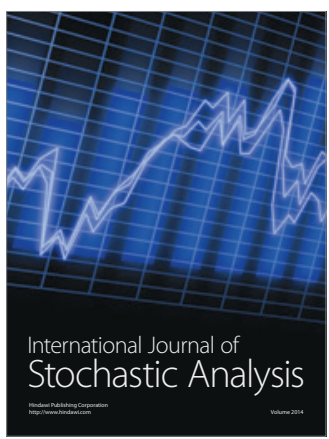

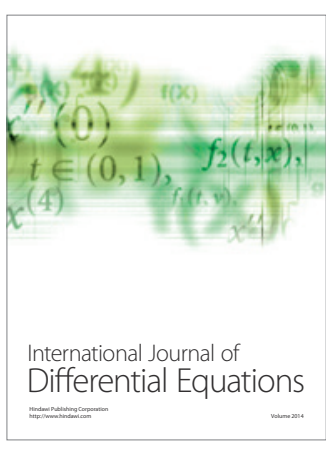
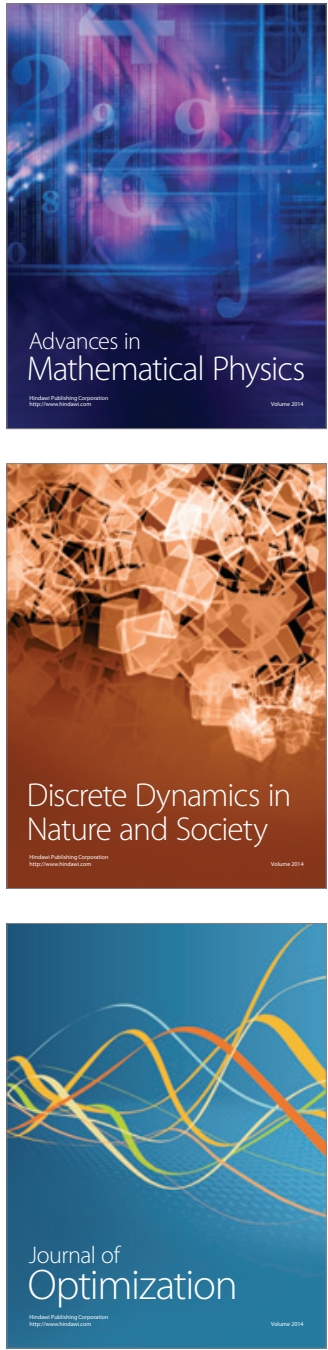